\title{
$L$-STRUCTURE IN $L$-SPACES $\left({ }^{1}\right)$
}

BY

F. CUNNINGHAM, JR.

1. Introduction. The concrete prototype of an $L$-space is the function space $L^{1}(S)$ of all summable real functions on a measure space $S$. Another model is the space $\mathfrak{R}(\mathcal{E})$ of all $\sigma$-additive functions (measures) on a Boolean $\sigma$-ring $\varepsilon$. It is easy to represent any $L^{1}(S)$ also as $\&(\varepsilon)$, taking in this case $\varepsilon$ to be the $\sigma$-ring of measurable sets in $S$ reduced modulo sets of measure 0 . It is less obvious that every $\mathcal{R}(\mathcal{E})$ is also representable as $L^{1}(S)$ for some measure space $S$, but Kakutani [8] gave such a representation for a whole class of Banach lattices called abstract L-spaces, which certainly includes the spaces $\mathfrak{R}(\mathcal{E})$ [3, p. 255]. Abstracting further, Fullerton [5] has given conditions on the shape of the unit sphere in a Banach space which are necessary and sufficient for the space to admit a partial ordering making it into an abstract $L$-space. Thus in the end $L$-spaces are Banach spaces of a certain kind.

The characterization theorems referred to are incomplete in this respect, that in each case the representation of the space in more concrete terms is not unique. In fact applying Kakutani's construction to the abstract $L$-space arising from $L^{1}(S)$ gives a new measure space not usually isomorphic to $S$. Similarly at another level, Fullerton's description relies on the existence of a cone having certain properties, and it is clear that an abstract $L$-space contains many such cones. Thus neither the measure space nor the partial order is an intrinsic feature of the Banach space; the representation is not canonical. The main purpose of this paper is to give a representation for $L$-spaces which is natural and, as far as possible, unique.

Let us call a measure $m$ on a complete Boolean algebra $\mathcal{F}$ completely additive (as opposed to merely $\sigma$-additive) when for any disjoint family $\left\{e_{\alpha}\right\}$ in $F$ of arbitrary cardinality

$$
m\left(\mathrm{~V}_{\alpha} e_{\alpha}\right)=\sum_{\alpha} m\left(e_{\alpha}\right)
$$

(equivalently, when for every increasing net $\left\{e_{\alpha}\right\}, m\left(\mathrm{~V}_{\alpha} e_{\alpha}\right)=\lim _{\alpha} m\left(e_{\alpha}\right)$ ). For a given algebra $F$, it is possible that no such measures exist, and we are interested only in the other extreme, namely complete algebras $\mathfrak{F}$ on which

Presented to the Society, December 29, 1953 under the title $L^{1}$-structure in Banach spaces. Preliminary report; received by the editors September 15, 1958 and, in revised form, April 29, 1959.

(1) The content of this paper is neither disjoint from nor contained in (nor does it wholly contain) the content of the author's hitherto unpublished doctoral thesis, Harvard University, 1953. The author wishes to express his indebtedness to Professor Lynn H. Loomis for his inspiration and guidance. 
enough completely additive measures exist to separate elements. Such algebras are exactly those whose Stone representation spaces are hyperstonian in the sense of Dixmier [4]. We propose to all them spectral algebras, because they are the abstract counterparts of complete Boolean algebras of projections.

Our representation theorem (Corollary 9.6) can now be stated as follows: any $L$-space is representable as the space $\mathfrak{R}(\mathcal{F})$ of all completely additive measures on a spectral algebra $\mathfrak{F}$, and the $\mathfrak{F}$ which will do this is (up to isomorphism) unique.

More generally, in any Banach space $\mathfrak{X}$ we shall call $L$-projections those projections which decompose $\mathfrak{X}$ into direct summands between which the norm is additive, as for example the projections in $L^{1}(S)$ associated with measurable sets in $S$. The set of all $L$-projections is, we find, a complete Boolean algebra of projections, hence abstractly a spectral algebra $\mathcal{F}$. The space is an $L$-space if and only if this " $L$-structure" is sufficiently rich, and when this is the case, $\mathcal{F}$ is the spectral algebra used in the representation.

The results of this paper overlap to some extent with those of Segal [12] and Dixmier [4], the former of these coming to the author's attention only after he had submitted the paper. These results are, however, simplified and enriched by being studied from our point of view emphasizing the $\sigma$-rings involved, and ignoring the measure theory (Segal) or topology (Dixmier) from which they may arise, or to which they give rise. Both Segal and Dixmier, each in his context, noted the connection between spectral algebras and spectral theory which motivates our terminology.

In $\S 2$ we show that the $L$-structure of any Banach space consists of a complete Boolean algebra of projections. Preliminary algebra having to do with Boolean rings is covered in $\$ 3$. We study completely additive measures in $\S 4$, the main result there being that the space $\&$ of all $\sigma$-additive measures on a $\sigma$-ring $\mathcal{E}$ splits into complementary $L$-subspaces $\mathfrak{N}$ and $\subseteq$ such that $\mathfrak{N}$ consists of all the completely additive measures on $\mathcal{E}$, while $\mathfrak{S}$ consists of the "purely $\sigma$-additive" measures. This decomposition is analogous to the decomposition of finitely additive measures into $\sigma$-additive and purely finitely additive parts [3, Theorem 17, p. 255] and [7]. Measure rings and spectral algebras, generalizing measure algebras, are defined in $\$ 5$, and in $\$ 6$ we extend to them Maharam's classification [11] of measure algebras. In view of Corollary 9.6 this gives a structure theorem for $L$-spaces.

$\S 7$ is a miniature integration theory, and $\$ \S 8$ and 9 are concerned with the related problems of conjugating $\Omega$ and of determining its $L$-structure. The main results here are the representation of $\mathcal{R}$ as $\mathfrak{R}(\mathfrak{F})$, where $\mathscr{F}$ is the $L$-decomposition of $\mathfrak{R}$, and the representation of $\Omega^{*}$ as the continuous function space on the Stone space of $\mathcal{F}$. This evidently solves each problem only in terms of the other, but in conjunction with the results of $\$ 2$ it can be regarded as a new description of $2^{*}$. In $\S 10$ we apply these results to the geometry of $L$ - 
spaces, obtaining a Banach space characterization of them and a description of their symmetries. Finally, in $\$ 11$ we mention a topological application in the form of a rearrangement of the proof of the Riesz theorem representing functionals on $C(\Sigma)$ ( $\Sigma$ compact and Hausdorff) by regular Borel measures.

There is reason to believe that the notion of $L$-structure should be useful in the theory of vector valued measures. In this direction the author's thesis (Harvard 1953) contains a canonical representation for an arbitrary Banach space as a direct integral on the Stone space of its $L$-decomposition, as well as a dual representation for its conjugate. Specializing to the "cylindrical" situation in which all the cross-sections are in an appropriate sense alike leads to problems about vector valued measures which we hope to deal with in another paper.

Another related question not touched on here is $L^{p}$-structure for $p>1$. In particular the case $p=\infty$, dual to $L$-structure, is of interest. Some preliminary results on this have been announced by the author (Abstract 365, Bull. Amer. Math. Soc. vol. 63 (1957) p. 191).

2. The $L$-decomposition of a Banach space. Let $\mathfrak{X}$ be a Banach space. A subspace $\mathfrak{X}_{1}$ of $\mathfrak{X}$ will be called an $L$-subspace if it has an $L$-complement, that is if there exists a subspace $\mathfrak{X}_{2}$ complementary to $\mathfrak{X}_{1}$ such that if $x_{1} \in \mathfrak{X}_{1}$ and $x_{2} \in \mathfrak{X}_{2}$, then $\left\|x_{1}+x_{2}\right\|=\left\|x_{1}\right\|+\left\|x_{2}\right\|$. Evidently any $L$-complement of an $L$ subspace is an $L$-subspace. It is easy to see that $L$-subspaces are closed. Indeed, suppose $\left\{x_{\alpha}\right\} \subset \mathfrak{X}_{1}$ and $\lim _{\alpha} x_{\alpha}=x_{0} \in \mathfrak{X}_{1}$. By subtracting from $x_{\alpha}$ the $\mathfrak{X}_{1}$-component of $x_{0}$, we can suppose $x_{0} \in \mathfrak{X}_{2}$. Then $\left\|x_{0}\right\|+\left\|x_{\alpha}\right\|=\left\|x_{0}-x_{\alpha}\right\|$ converges to 0 , whence $x_{0}=0$.

\section{LemMa 2.1. The L-complement of an L-subspace is unique.}

Proof. Suppose $\mathfrak{X}_{2}$ and $\mathfrak{X}_{3}$ are $L$-complements of $\mathfrak{X}_{1}$. Let $x_{3} \in \mathfrak{X}_{3}$. Then $x_{3}=x_{1}+x_{2}$, where $x_{1} \in \mathfrak{X}_{1}$ and $x_{2} \in \mathfrak{X}_{2}$, and $\left\|x_{3}\right\|=\left\|x_{1}\right\|+\left\|x_{2}\right\|=\left\|x_{1}\right\|+\left\|-x_{1}\right\|$ $+\left\|x_{3}\right\|$, whence $x_{1}=0$ and $x_{3} \in \mathfrak{X}_{2}$. We have shown $\mathfrak{X}_{3} \subset \mathfrak{X}_{2}$, which by symmetry gives $\mathfrak{X}_{3}=\mathfrak{X}_{2}$.

By virtue of this lemma any $L$-subspace determines a unique projection having it as range and annihilating its $L$-complement. Such projections will be called $L$-projections. In particular 0 and the identity 1 are $L$-projections, and of course there may be no others. The class $\mathcal{F}=\mathfrak{F}(\mathfrak{X})$ of all $L$-projections in $\mathfrak{X}$ will be called the $L$-decomposition of $\mathfrak{X}$. For a projection $E$ we write $E \mathfrak{X}$ for its range; its null-space is then $(1-E) \mathfrak{X}$. Any nonzero projection automatically has norm at least 1 , but $L$-projections have norm 1 , since $\|x\|$ $=\|E x\|+\|(1-E) x\| \geqq\|E x\|$ for all $x$.

\section{LEMMA 2.2. All L-projections commute.}

Proof. Let $E$ and $E^{\prime}$ be arbitrary $L$-projections. Resolving $E x$ first into $E^{\prime}$ and $1-E^{\prime}$ components, then into $E$ and $1-E$ components gives 


$$
\begin{aligned}
\|E x\| & =\left\|E^{\prime} E x\right\|+\left\|\left(1-E^{\prime}\right) E x\right\| \\
& =\left\|E E^{\prime} E x\right\|+\left\|(1-E) E^{\prime} E x\right\|+\left\|E\left(1-E^{\prime}\right) E x\right\|+\left\|(1-E)\left(1-E^{\prime}\right) E x\right\| .
\end{aligned}
$$

On the other hand, $E x=E E^{\prime} E x+E\left(1-E^{\prime}\right) E x$, whence

$$
\|E x\| \leqq\left\|E E^{\prime} E x\right\|+\left\|E\left(1-E^{\prime}\right) E x\right\| .
$$

Comparing the right members reveals that

$$
(1-E) E^{\prime} E x=(1-E)\left(1-E^{\prime}\right) E x=0,
$$

whence $E^{\prime} E=E E^{\prime} E$. Replacing $E$ by $1-E$ gives $E^{\prime}(1-E)=(1-E) E^{\prime}(1-E)$, or $E E^{\prime}=E E^{\prime} E$. Hence $E^{\prime} E=E E^{\prime}$.

Lemma 2.3. If $E$ and $E^{\prime}$ are L-projections, then $E E^{\prime}$ is an L-projection.

Proof. That $E E^{\prime}$ is a projection follows from 2.2. Now

$$
\begin{aligned}
\left(1-E E^{\prime}\right) x & =E\left(1-E E^{\prime}\right) x+(1-E)\left(1-E E^{\prime}\right) x \\
& =E\left(1-E^{\prime}\right) x+(1-E) x
\end{aligned}
$$

whence $\left\|\left(1-E E^{\prime}\right) x\right\|=\left\|E\left(1-E^{\prime}\right) x\right\|+\|(1-E) x\|$. Thus

$$
\begin{aligned}
\|x\| & =\left\|E^{\prime} x\right\|+\left\|\left(1-E^{\prime}\right) x\right\| \\
& =\left\|E E^{\prime} x\right\|+\left\|(1-E) E^{\prime} x\right\|+\left\|E\left(1-E^{\prime}\right) x\right\|+\left\|(1-E)\left(1-E^{\prime}\right) x\right\| \\
& =\left\|E E^{\prime} x\right\|+\left\|E\left(1-E^{\prime}\right) x\right\|+\|(1-E) x\| \\
& =\left\|E E^{\prime} x\right\|+\left\|\left(1-E E^{\prime}\right) x\right\| .
\end{aligned}
$$

LemMa 2.4. If $\left\{E_{\alpha}\right\}$ is a nondecreasing net of $L$-projections then $\left\{E_{\alpha}\right\}$ converges strongly to an L-projection.

Proof. The partial order $E_{\alpha} \leqq E_{\beta}$ refers, of course, to the inclusion $E_{\alpha} \mathfrak{X} \subset E_{\beta} \mathfrak{X}$. Now, $\left\{E_{\alpha}\right\}$ being nondecreasing, if $\alpha \leqq \beta$, then for any $x$

$$
\begin{aligned}
\|x\| & \geqq\left\|E_{\beta} x\right\| \\
& =\left\|E_{\alpha} x\right\|+\left\|\left(E_{\beta}-E_{\alpha}\right) x\right\| \\
& \geqq\left\|E_{\alpha} x\right\| .
\end{aligned}
$$

Therefore $\left\{\left\|E_{\alpha} x\right\|\right\}$ is a nondecreasing net of real numbers bounded above by $\|x\|$, hence convergent. We can use this to show that $\left\{E_{\alpha} x\right\}$ is a Cauchy net in $\mathfrak{X}$. Indeed, given $\epsilon>0$ choose $\alpha$ so that $\left\|E_{\alpha} x\right\| \geqq \lim _{\beta}\left\|E_{\beta} x\right\|-\epsilon / 2$. Then $\alpha \leqq \beta$ and $\alpha \leqq \beta^{\prime}$ imply

$$
\begin{aligned}
\left\|E_{\beta} x-E_{\beta^{\prime}} x\right\| & \leqq\left\|E_{\beta} x-E_{\alpha} x\right\|+\left\|E_{\beta^{\prime}} x-E_{\alpha} x\right\| \\
& =\left\|E_{\beta} x\right\|-\left\|E_{\alpha} x\right\|+\left\|E_{\beta^{\prime}} x\right\|-\left\|E_{\alpha} x\right\| \\
& \leqq 2 \lim _{\beta}\left\|E_{\beta} x\right\|-2\left\|E_{\alpha} x\right\| \\
& \leqq \epsilon .
\end{aligned}
$$


Since the limit of this convergent net depends linearly on $x$ and its norm does not exceed $\|x\|$, we write it $E_{0} x$, where $E_{0}$ is an operator. Now as soon as $\beta \geqq \alpha$ we have $E_{\alpha} E_{\beta}=E_{\alpha}$, whence the continuity of $E_{\alpha}$ gives $E_{\alpha} E_{0} x=\lim _{\beta} E_{\alpha} E_{\beta} x$ $=\lim _{\beta} E_{\alpha} x=E_{\alpha} x$. Thus $E_{0}^{2} x=\lim _{\alpha} E_{\alpha} E_{0} x=E_{0} x$, and $E_{0}$ is a projection. Finally

$$
\begin{aligned}
\left\|E_{0} x\right\|+\left\|\left(1-E_{0}\right) x\right\| & =\lim _{\alpha}\left(\left\|E_{\alpha} x\right\|+\left\|\left(1-E_{\alpha}\right) x\right\|\right) \\
& =\|x\|,
\end{aligned}
$$

so that $E_{0}$ is an $L$-projection.

A Boolean algebra $F$ of projections is said to be complete [1] when any family $\left\{E_{\alpha}\right\}$ in $\mathscr{F}$ has a supremum in $\mathcal{F}$ whose range is the closed linear span of the $E_{\alpha} \mathfrak{X}$, and whose null-space is the intersection of the $\left(1-E_{\alpha}\right) \mathfrak{X}$.

Theorem 2.5. The L-decomposition $\mathfrak{F}(\mathfrak{X})$ of a Banach space $\mathfrak{X}$ is a complete Boolean algebra of commuting projections.

Proof. Lemmas 2.2 and 2.3 show that $\mathcal{F}$ is a Boolean algebra. To show that $\mathcal{F}$ is complete, let $\left\{E_{\alpha}\right\}$ be a family of $L$-projections. Then the set of finite joins of projections $\left\{E_{\alpha}\right\}$ gives the same closed linear span and has the Moore-Smith property upward. Considered as a nondecreasing net it converges strongly to an $L$-projection $E_{0}$ by 2.4 , which satisfies the requirements for the supremum of $\left\{E_{\alpha}\right\}$, as is easy to verify.

The completeness of $\mathcal{F}(\mathfrak{X})$ of course implies its completeness as an abstract Boolean algebra. Moreover there are plenty of completely additive measures on it. For any $x$, let $E_{0}$ be the meet of all $L$-projections whose ranges contain $x$. Then by Lemma $2.4\|E x\|$ as a function of $E$ is a completely additive measure on $\mathscr{F}(\mathfrak{X})$, which is positive on the principal ideal generated by $E_{0}$, and 0 on its complement. Any nonzero $E$ in $\mathscr{F}$ contains in its range a nonzero $x$, and then $E_{0} \neq 0$ and $E_{0} \leqq E$.

3. The compactification of a $\sigma$-ring. Any abstract Boolean ring $\mathcal{E}$ can be realized by Stone's construction [13] as the ring of open compact sets in a locally compact Boolean space $\Omega=\Omega(\mathcal{E})$ in which the family $\mathcal{E}$ is a basis for the topology. To minimize notation we shall make no distinction between the elements $e$ of $\mathcal{E}$ and the subsets of $\Omega$ which represent them. The reader is warned, however, that while finite meets and joins in $\varepsilon$ become set intersections and unions, this is not so for infinite ones when they exist. The formulas are in fact

$$
\begin{aligned}
& \bigwedge_{\alpha} e_{\alpha}=\operatorname{Int} \bigcap_{\alpha} e_{\alpha}, \\
& \bigvee_{\alpha} e_{\alpha}=\operatorname{Cl~}_{\alpha} e_{\alpha},
\end{aligned}
$$

where $\Lambda$ and $V$ represent infimum and supremum in $\varepsilon$, while $\cap$ and $U$ represent set operations. Indeed, if for example $e_{0}=\Lambda_{\alpha} e_{\alpha}$ exists in $\varepsilon$, then $e_{0}$ being open and contained in each $e_{\alpha}$, we have $e_{0} \subset \operatorname{Int} \bigcap_{\alpha} e_{\alpha}$. On the other hand, $e_{0}$ being closed, Int $\bigcap_{\alpha} e_{\alpha}-e_{0}$ is open, and if not empty must contain some open 
compact $e_{1} \neq 0$. Then $e_{0} \vee e_{1}$ is contained in every $e_{\alpha}$, so that $e_{0}$ cannot be the infimum. The dual formula is proved similarly.

Suppose now that $\varepsilon$ is a Boolean $\sigma$-ring. Let $\beta$ be the $\sigma$-ring of sets generated by $\mathcal{E}$, and let $\Re$ be the $\sigma$-ideal in $\Theta$ generated by sets of the form $\bigcap_{n=1}^{\infty} e_{n}$ $-\Lambda_{n=1}^{\infty} e_{n}$. It is easy to see that every element of $B / \mathcal{N}$ contains a set of $\varepsilon$, and therefore that the restriction to $\mathcal{E}$ of the natural homomorphism of $B$ on $B / \mathscr{N}$ maps $\mathcal{E}$ onto $B / \mathscr{T}$. According to the theorem of Loomis [10] this map is a $\sigma$-isomorphism.

The effect on $\Omega$ of passing from $\varepsilon$ to a subring or a homomorphic image are found in [13]. Suppose $g$ is a subring of $\mathcal{E}$, and let $\beta$ be the identity map of $\mathscr{g}$ into $\mathcal{E}$. Define a continuous map $\phi$ of the open set $U \mathscr{g}=U_{e \in \mathscr{g}} e$ into $\Omega(\mathfrak{g})$ by making $\phi(x)$ the unique point in $\Omega(\mathscr{g})$ belonging to the intersection of all $\beta^{-1}(e)$ such that $x \in e$. Then $\phi$ maps $U \mathscr{g}$ continuously onto $\Omega(\mathscr{g})$, and for every $e$ in $g, \beta(e)=\phi^{-1}(e)$. It is easy to show that $\phi$ is a homeomorphism if and only if $\mathscr{g}$ is an ideal.

If $\mathscr{g}$ is an ideal in $\mathcal{E}$, and if $\alpha$ is the natural homomorphism of $\varepsilon$ onto $a=\varepsilon / g$, define $\psi$ mapping $\Omega(\alpha)$ into $\Omega(\mathcal{E})$ by making $\psi(x)$ the unique point in $\Omega(\varepsilon)$ belonging to the intersection of all sets $e$ such that $x \in \alpha(e)$. Then $\psi$ is a homeomorphism of $\Omega(\mathfrak{Q})$ onto the closed set $C(\mathcal{g})$ in $\Omega(\mathcal{E})$ complementary to Ug.

We write $e \wedge \mathcal{E}$ for the principal ideal generated by $e$, and $\mathscr{g}^{\perp}$ ( $\mathscr{g}$ being any subset of $\mathcal{E}$ ) for the ideal $\left\{e: e^{\prime} \in \mathscr{g}\right.$ implies $\left.e \wedge e^{\prime}=0\right\}$. An ideal $g$ is called dense when $\mathfrak{g}^{\perp}=0$.

We shall be interested in the space $\subseteq(\Omega)$ of all bounded continuous real functions on $\Omega$. If $\mathcal{E}$ is a Boolean algebra, so that $\Omega$ is compact, then by the Stone-Weierstrass theorem $\mathfrak{S}(\Omega)$ is generated by linear combination and uniform closure from the characteristic functions of sets belonging to $\mathcal{E}$. Consider now the noncompact case. The class $\bar{\varepsilon}$ of open closed sets in $\Omega$ is a Boolean algebra having $\mathcal{E}$ as a dense ideal. Thus $\Omega$ is (homeomorphic to, hence identified with) a dense open set in $\bar{\Omega}=\Omega(\overline{\mathcal{E}})$. We shall see that when $\mathcal{E}$ is a $\sigma$-ring, $\bar{\Omega}$ is the Stone-Čech compactification of $\Omega$, and we therefore refer to $\bar{\varepsilon}$ as the compactification of $\varepsilon$.

Lemмa 3.2. If $\mathcal{E}$ is a $\sigma$-ring, then every closed $G_{\delta}$ in $\Omega$ has a closed interior, and the Boolean algebra $\bar{\varepsilon}$ of open closed sets in $\Omega$ is a $\sigma$-algebra.

Proof. Since a subset of $\Omega$ is closed if and only if its intersection with every open compact set is closed, and since for any set $A$ and any open compact set $e, \operatorname{Int}(e \cap A)=e \bigcap \operatorname{Int} A$, it suffices to show that the interior of any compact $G_{\delta}$ is closed. But if $A$ is a compact $G_{\delta}, \varepsilon$ being a basis for the topology, it is easy to see that $A=\bigcap_{n=1}^{\infty} e_{n}$ for a suitable sequence $\left\{e_{n}\right\}$ in $\mathcal{E}$, and then according to 3.1 Int $A=\Lambda_{n=1}^{\infty} e_{n}$, which is closed. Finally, to see that $\bar{\varepsilon}$ is a $\sigma$-algebra, let $\left\{u_{n}\right\}$ be a sequence in $\bar{\varepsilon}$. Then $\bigcap_{n=1}^{\infty} u_{n}$ is a closed $G_{\delta}$, whence Int $\bigcap_{n=1}^{\infty} u_{n} \in \bar{\varepsilon}$ and is the infimum of $\left\{u_{n}\right\}$. 
THEOREM 3.3. If $\&$ is a $\sigma$-ring, then every bounded continuous function on $\Omega$ has a continuous extension to $\bar{\Omega}$.

Proof. Continuous characteristic functions extend trivially. Since the class of functions which have extensions is closed under the linear operations and uniform convergence, we need only show that any bounded continuous function on $\Omega$ can be uniformly approximated by linear combinations of continuous characteristic functions. Given $f \in \mathbb{E}(\Omega)$ and $\epsilon>0$, cover the range of $f$ by closed intervals $I_{1}, \cdots, I_{n}$, disjoint except for common end-points, each of length less than $\epsilon$. For each $i=1, \cdots, n, f^{-1}\left(I_{i}\right)$ is a closed $G_{\delta}$, and by 3.2 its interior $u_{i}$ is open and closed. Moreover in $u_{i}, f$ has only values in $I_{i}$, while outside $u_{i}, f$ has no values in the interior of $I_{i}$. Readjust $u_{1}, \cdots, u_{n}$ in the obvious way to make them disjoint and take a suitable linear combination of their characteristic functions to obtain a function uniformly within $\epsilon$ of $f$.

In the correspondence between open sets in $\Omega$ and ideals in $\mathcal{E}$, the open closed sets correspond to locally principal ideals, that is ideals $\mathscr{g}$ such that $g \cap(e \wedge \varepsilon)$ is principal for every $e$. It is clear that locally principal ideals are not only $\sigma$-ideals, but even closed in the sense of satisfying the equivalent conditions (1) if $\left\{e_{\alpha}\right\} \subset \mathcal{g}$ and $\mathrm{V}_{\alpha} e_{\alpha}$ exists then $\mathrm{V}_{\alpha} e_{\alpha} \in \mathcal{G}$, and (2) $\mathfrak{g}^{\perp \perp}=\mathfrak{g}$. If $\mathcal{E}$ happens to be boundedly complete, then every closed ideal is locally principal, and $\bar{\varepsilon}$ is just the usual completion of $\mathcal{\varepsilon}$. We state this for reference.

Lemma 3.4. If $\mathcal{E}$ is boundedly complete, then $\bar{\varepsilon}$ is complete; conversely, if $\mathcal{F}$ is any complete Boolean algebra, then $\mathcal{F}$ is the compactification of each of its dense $\sigma$-ideals.

Proof. The proof of the first part is just like that of 3.2. For the second part observe that the relation between elements of $\bar{\varepsilon}$ and locally principal ideals in $\varepsilon$ is this: to each $u$ in $\bar{\varepsilon}$ corresponds the locally principal ideal $\mathfrak{I}_{u}=\{e \in \mathcal{E}: e \leqq u\}$ in $\mathcal{E}$. Therefore, given a complete Boolean algebra $\mathfrak{F}$, and a dense $\sigma$-ideal $\mathcal{E}$ in $\mathcal{F}$, it suffices to prove that applying this formula to elements of $\mathscr{F}$ gives all locally principal ideals in $\mathcal{E}$. That $\mathscr{g}_{u}$ is locally principal is clear. On the other hand, let $g$ be a locally principal ideal in $\mathcal{E}$, and let $u=\mathrm{V}\{e: e \in \mathfrak{I}\}$ (in $\mathcal{F}$ ). We must show that $\mathscr{I}_{u} \subset \mathcal{G}$. Now if $e \in \mathcal{E}$, and $e \leqq u$, then $\mathfrak{g}$ is dense in $e \wedge \mathcal{E}$. Therefore, since $\mathscr{g}$ is locally principal, $\mathscr{g} \cap(e \wedge \mathcal{E})=e \cap \mathcal{E}$, that is $e \in g$.

4. Normal measures. By a measure on a $\sigma$-ring $\mathcal{E}$ we shall mean, with two exceptions duly noted, a real finite-valued $\sigma$-additive function defined on $\mathcal{E}$. Denote the class of these by $\mathfrak{l}=\mathfrak{R}(\mathcal{E})$. Every measure $m$ is bounded and has positive and negative variations $m^{+}$and $m^{-}$in $\&$, which are non-negative, satisfy $m=m^{+}-m^{-}$, and are defined by

$$
\begin{aligned}
& m^{+}(e)=\sup \left\{m\left(e^{\prime}\right): e^{\prime} \leqq e\right\}, \\
& m^{-}(e)=-\inf \left\{m\left(e^{\prime}\right): e^{\prime} \leqq e\right\} .
\end{aligned}
$$


Denote by $|m|$ the total variation $m^{+}+m^{-}$. Because $|m|$ is $\sigma$-additive, it assumes a maximum value on some $e$ in $\mathcal{E}$, and therefore vanishes on $(e \wedge \mathcal{E})^{\perp}$. Such an $e$ (not unique) we call a container for $m$. The norm

$$
\begin{aligned}
\|m\| & =\sup \{|m|(e)\} \\
& =|m|(e) \quad(e \text { a container })
\end{aligned}
$$

makes $\mathbb{R}$ into a Banach space, in fact an abstract $L$-space [3, p. 255].

The set of those $e$ such that $m$ vanishes on $e \wedge \mathcal{E}$ (equivalently, such that $|m|(e)=0)$ is a $\sigma$-ideal $\mathscr{g}_{m}$. The set $S_{m}$ complementary to $U \mathscr{I}_{m}$ in $\Omega$ we shall call the support of $m$. Because of the existence of containers supports are compact. Clearly $S_{|m|}=S_{m}$, and $S_{m}$ contains the supports of $m^{+}$and $m^{-}$. When the equivalent conditions $S_{m_{1}} \subset S_{m_{2}}$ or $\mathscr{I}_{m_{2}} \subset \mathfrak{g}_{m_{1}}$ hold for measures $m_{1}$ and $m_{2}$ we say that $m_{1}$ is absolutely continuous with respect to $m_{2}$ and write $m_{1} \ll m_{2}$.

Some $L$-structure in $\mathcal{R}$ is immediately apparent, associated with the compactification $\bar{\varepsilon}$ of $\mathcal{E}$. In fact any $u$ in $\bar{\varepsilon}$ determines a projection $\tilde{u}$ operating on $\mathcal{R}$ as follows: $\tilde{u} m(e)=m(u \wedge e)(u \wedge e$ belonging to $\mathcal{E}$ because $\mathcal{E}$ is an ideal). Clearly $\tilde{u} m^{+}=(\tilde{u} m)^{+}$and $\tilde{u} m^{-}=(\tilde{u} m)^{-}$, so $\tilde{u}|m|=|\tilde{u} m|$. Hence for a container $e$ of $m$ we have

$$
\begin{aligned}
\|m\|=|m|(e) & =|m|(u \wedge e)+|m|((1-u) \wedge e) \\
& =|\tilde{u} m|(e)+|m-\tilde{u} m|(e) \\
& =\|\tilde{u} m\|+\|(1-\tilde{u}) m\|
\end{aligned}
$$

and $\tilde{u}$ is an $L$-projection. We shall now generalize this situation.

Lemma 4.1. Let $g$ be a $\sigma$-ideal in $\mathcal{E}$. Let $\mathfrak{X}$ be the set of all $m$ in $\mathfrak{R}$ whose supports are contained in $\cup g$, and let $\mathfrak{Y}$ be the set of all $m$ which vanish on $\mathfrak{g}$. Let $\alpha$ be the natural homomorphism of $\mathcal{E}$ on $a=\varepsilon / g$. Then:

(1) the mapping $m \rightarrow T m$, where $\operatorname{Tm}(e)=m(\alpha e)$ for $m$ in $\mathfrak{R}(Q)$, is an isometry of $\mathbb{R}(\mathbb{Q})$ on $\mathfrak{Y}$;

(2) restriction to $\mathfrak{g}$ is an isometry of $\mathfrak{X}$ onto $\mathfrak{R}(\mathfrak{g})$;

(3) $\mathfrak{X}$ and $\mathfrak{Y}$ are complementary $L$-subspaces of $\mathfrak{R}$.

Proof. (1) Since $T$ preserves non-negativeness, $T|m|=|T m|$. Let $e \in \mathcal{E}$ be such that $\alpha e$ is a container for $m$. Then $e$ is a container of $T m$, and $\|T m\|$ $=|T m|(e)=|m|(\alpha e)=\|m\|$. Thus $T$ is an isometry. Clearly $T$ maps $\mathfrak{R}(\mathbb{Q})$ into $\mathfrak{Y}$, since $\alpha$ maps $g$ into 0 . Moreover any measure on $\varepsilon$ which vanishes on $\mathscr{I}$ is constant on sets which belong to $\mathcal{E} / \mathscr{G}$, and is therefore expressible as $m(\alpha e)$ for some $m$ in $\mathscr{R}(\alpha)$.

(2) Suppose $m \in \mathfrak{X}$. Then $S_{m} \subset U_{g}$, and using the compactness of $S_{m}$, $S_{m} \subset e \subset U_{g}$ for some $e$, which is then a container for $m$ belonging to $\mathscr{g}$. The norm of the restriction of $m$ to $g$ is then $|m|(e)=\|m\|$. That restriction gives all of $\mathfrak{R}(\mathfrak{g})$ amounts to the possibility of extending to $\mathcal{E}$ any measure on $\mathscr{g}$. 
This is done simply by making $m$ vanish on $(e \wedge \varepsilon)^{\perp}$, where $e$ is a container for $m$ in $g$.

(3) It is clear that $\mathfrak{X} \cap \mathfrak{Y}=0$. Now for any $m$ in $L$, let $e$ be a container in $\mathscr{g}$ for the restriction of $m$ to $\mathscr{g}$. Then $\tilde{e} m \in \mathfrak{X}$, and $m-\tilde{e} m \in \mathfrak{Y}$, and $\|m\|=\|\tilde{e} m\|$ $+\|m-\tilde{e} m\|$.

Corollary 4.2 (Lebesgue decomposition). For any $m$ in $\&$, the set of measures absolutely continuous with the respect to $m$ and the set of measures whose supports are disjoint from $S_{m}$ are complementary L-subspaces in $\&$.

REMARK. If $\mathscr{g}$ is locally principal, 4.1 gives the same projection as was described in the preceding discussion. There can be $\sigma$-ideals, however, which are not locally principal (for example, $\mathscr{g}$ could be the ideal of all countable sets in $\mathcal{E}$, the algebra of all subsets of an uncountable universe). On the other hand 4.1 is not definitive: there can be $L$-projections not arising from any $\sigma$-ideals. Indeed, if $E$ is the $L$-projection arising from $g$ in the example just mentioned, then $1-E$ corresponds to no $\sigma$-ideal. Evidently the combination of two such examples suffices to show the possibility of neither $E$ nor $1-E$ arising in this way.

Lemma 4.3. The set of elements of $\mathcal{E}$ which are supports of measures is a $\sigma$-ideal.

Proof. Note that $e$ is the support of $m$ if and only if $|m|$ vanishes on $(e \wedge \varepsilon)^{\perp}$ and is strictly positive on $e \wedge \mathcal{E}$. Thus if $e$ is the support of $m$, and $e^{\prime} \leqq e$, then $e^{\prime}$ is the support of $\tilde{e}^{\prime} m$, while if for each positive integer $n, e_{n}$ is the support of $m_{n}$, and $e=\mathrm{V}_{n} e_{n}$, then $e$ is the support of $\sum_{n} 2^{-n} m_{n}$.

If $\mathcal{E}$ is a measure algebra, a well known procedure, the method of exhaustion can be used to prove that any $\sigma$-ideal $g$ is principal. To obtain the generator of $\mathscr{g}$ one takes $\mathrm{V}_{n=1}^{\infty} e_{n}$, where $\left\{e_{n}\right\}$ is a sequence in $\mathscr{I}$ with $m\left(e_{n}\right)$ increasing to $\sup \{m(e): e \in \mathcal{I}\}, m$ being a finite strictly positive measure on $\varepsilon$. One consequence of this is that all measures on a measure algebra have open supports, a fact generalized in the next theorem.

THEOREM 4.4. Let $\mathfrak{X}$ be the set of measures in $\mathbb{R}$ having open supports, and let $\mathfrak{Y}$ be the set of measures having nondense supports. Then the supports of measures in $\mathfrak{X}$ form a $\sigma$-ideal $\mathcal{E}_{N}$; restriction to $\mathcal{E}_{N}$ is an isometry of $\mathfrak{X}$ on $\mathfrak{R}\left(\mathcal{E}_{N}\right) ; \mathfrak{Y}$ consists of all measures which vanish on $\mathcal{E}_{N}$; and $\mathfrak{X}$ and $\mathfrak{Y}$ are complementary L-subspaces of $\mathrm{R}$.

Proof. If we establish that $\mathfrak{X}$ consists of all measures with supports in $\cup \mathcal{E}_{N}$ and $\mathfrak{Y}$ of all measures which vanish on $\mathcal{E}_{N}$, then the theorem will follow immediately from 4.1 and 4.3. Suppose $m$ has its support contained in $U \mathcal{E}_{N}$. Then $m$ has a container $e$ in $\varepsilon_{N}$, and $e$ is the support of a measure $m_{0}$. This means that $e \wedge \mathcal{E}$ is a measure algebra, and by the remark preceding the theorem, $m$ has an open support contained in $e$. 
Now let $m \in \mathfrak{Y}$. If for some $e$ in $\mathcal{E}_{N}|m|(e) \neq 0$, then by what we have already proved $\tilde{e} m \in \mathfrak{X}$, and the support of $\tilde{e} m$ is an open set contained in the support of $m$, a contradiction. On the other hand, if the support of $m$ is not nondense, it contains a nonzero open compact set $e$. Then $e$ is the support of $\tilde{e} m$, hence belongs to $\varepsilon_{N}$, and $m$ does not vanish on $\varepsilon_{N}$. This completes the proof.

Lemma 4.5 (Dixmier [4]). The support of $m$ is open if and only if $m$ is completely additive.

Proof. Suppose first that the support of $m$ is open. Since the supports of $m^{+}$and $m^{-}$are contained in that of $m$, they are open by 4.4, and we need consider only $m \geqq 0$. By going down to a principal ideal we can suppose the support of $m$ is 1 and it is enough to treat $\left\{e_{\alpha}\right\}$ such that $V_{\alpha} e_{\alpha}=1$. From $\left\{e_{\alpha}\right\}$ choose an increasing sequence $\left\{e_{n}\right\}$ such that $\lim _{n} m\left(e_{n}\right)=\sup _{\alpha} m\left(e_{\alpha}\right)=k$, and let $e_{0}=\mathrm{V}_{n} e_{n}$. Then $m\left(e_{0}\right)=k$, because $m$ is $\sigma$-additive. Now $e_{0} \neq 1$ would lead to the existence of an $e_{\alpha}$ with $m\left(e_{\alpha}\right)>k$ (because $m$ is strictly positive, having 1 for support), which is impossible. Thus $m(1)=k=\lim _{\alpha} m\left(e_{\alpha}\right)$.

Conversely, suppose $m$ is completely additive. Using 4.4 we write $m$ as $m_{N}+m_{S}$, where $m_{N}$ has an open support, and $m_{S}$ a nondense support. Then $m$ and $m_{N}$ (by the first part of the proof) both being completely additive, $m_{S}$ is also. It thus suffices to show that any completely additive $m$ with nondense support must vanish identically. For any $e$ in $\mathcal{E}$ the ideal $\mathscr{g}=\mathscr{I}_{m} \cap(e \wedge \mathcal{E})$ has $e$ for supremum. For if $e^{\prime} \leqq e, e^{\prime} \neq e$, then $e-e^{\prime}$, being a nonempty open set, is not contained in the support of $m$, so intersects some element of $g_{m}$, and therefore $e^{\prime}$ is not an upper bound for $\mathscr{g}$. The complete additivity of $m$ and its vanishing on $\mathscr{g}$ give $m(e)=0$, and since $e$ was arbitrary, $m=0$.

For brevity and to avoid misunderstanding, we shall call measures which are completely additive in our sense, or equivalently which have open supports, normal measures, following the terminology of Dixmier. Measures which have nondense supports, by contrast, we shall call purely $\sigma$-additive. Note that $m$ is purely $\sigma$-additive if and only if for any normal measure $m^{\prime}, 0 \leqq m^{\prime} \leqq|m|$ implies $m^{\prime}=0$.

Denote by $\mathfrak{N}=\mathfrak{N}(\mathcal{E})$ the space of all normal measures on $\mathcal{\varepsilon}$. The $\sigma$-ideal $\varepsilon_{N}$ consisting of supports of measures in $\mathfrak{N}$ will be called the normal ideal of $\varepsilon$. From 4.4 we have $\mathfrak{R}(\mathcal{E})=\mathfrak{R}\left(\varepsilon_{N}\right)$.

5. Measure rings and spectral algebras. A measure algebra is distinguished by the existence of a strictly positive finite measure on it. We develop now two rather superficial generalizations of measure algebra aimed at removing the restriction on size inherent in the finiteness of the measure. Like measure algebra, these generalizations have definitions which are not algebraic, but rather rely on the existence of positive measures. For this purpose, and here only, we have to admit measures having infinite values. 
THEOREM 5.1. The following conditions on a $\sigma$-ring $\mathcal{E}$ are equivalent:

(1) $\varepsilon_{N}=\varepsilon$

(2) every principal ideal is a measure algebra;

(3) there exists on $\&$ a strictly positive $\sigma$-finite measure.

Proof. The equivalence of (1) and (2) is obvious. Assume (3). If $m$ is positive and $\sigma$-finite on $\mathcal{E}$ and $e \in \mathcal{E}$, then $e=\bigvee_{n=1}^{\infty} e_{n}$, where $m\left(e_{n}\right)<\infty$. Then $\sum_{n=1}^{\infty} 2^{-n} \tilde{e}_{n} m$ is a strictly positive finite measure on $e \wedge \mathcal{E}$, proving (2). Finally, assume (2). Choose a maximal family $\left\{m_{\alpha}\right\}$ in $\&$ such that $m_{\alpha} \geqq 0$, their supports $\left\{e_{\alpha}\right\}$ are disjoint, and $m_{\alpha}\left(e_{\alpha}\right)=1$. Define a $\sigma$-finite measure on $\mathcal{E}$ as follows: any $e$ in $\mathcal{E}$, being the support of a finite measure, intersects only a countable subfamily $\left\{e_{n}\right\}$ of $\left\{e_{\alpha}\right\}$, and by the maximality of $\left\{m_{\alpha}\right\}$, $e=\mathrm{V}_{n}\left(e_{n} \wedge e\right)$; define $m(e)$ as $\sum_{n} m\left(e_{n} \wedge e\right)$. Clearly $m$ is strictly positive, proving (3).

A $\sigma$-ring satisfying the conditions (1)-(3) of 5.1 we shall call a measure ring. The $\sigma$-ring $\mathfrak{M} / \mathfrak{M}_{0}$ associated with a measure space (see $\S 1$ ) is a measure ring. Conversely, condition (3) shows that every measure ring can be so realized, by taking $\mathfrak{T}$ as the ring of Baire sets in $\Omega$. Thus for measure rings the representation of $\&$ as $L^{1}$ is immediate. Evidently a measure ring is a measure algebra if and only if it has a unit. The following properties of measure rings generalize properties of measure algebras.

Theorem 5.2. If $\mathcal{E}$ is a measure ring, then (1) $\mathcal{E}$ is boundedly complete, (2) every measure on $\mathcal{E}$ is normal, and (3) every $\sigma$-ideal is locally principal.

Proof. (1) is immediate from the fact that measure algebras are complete, and (2) follows from 4.4. To prove (3), let $\mathfrak{g}$ be a $\sigma$-ideal, and let $\mathfrak{g}_{e}=\mathfrak{g} \cap(e \wedge \mathcal{E})$. By hypothesis $e$ is the support of some $m \geqq 0$. Choose an increasing sequence $\left\{e_{n}\right\}$ in $\mathscr{g}_{e}$ such that $\lim _{n} m\left(e_{n}\right)=\sup \left\{m\left(e^{\prime}\right): e^{\prime} \in \mathscr{g}_{e}\right\}$. Then $e_{0}=\mathrm{V}_{n} e_{n} \in \mathscr{I}_{e}$ and $m\left(e_{0}\right)$ is maximal. Hence by the positivity of $m$ on $e \wedge \mathcal{E}, \mathscr{I}_{e}$ is the principal ideal $e_{0} \wedge \mathcal{E}$, q.e.d.

A measure $m$ will be called locally finite if for any nonzero $e$ there exists a nonzero $e^{\prime} \leqq e$ such that $m\left(e^{\prime}\right)<\infty$. Of course $\sigma$-finite measures are locally finite. Every $\sigma$-ring has a strictly positive measure which is not locally finite (define $m(e)$ to be $\infty$ except for $e=0$ ), and for any measure $m$ whatever, $\mathcal{E}$ splits into two disjoint closed ideals on one of which $m$ is locally finite, and on the other identically infinite (like the parenthetical example).

THEOREM 5.3. The following conditions on a complete Boolean algebra $\mathfrak{F}$ are equivalent:

(1) $\mathfrak{F}_{N}$ is dense;

(2) $\mathcal{F}$ is the compactification of a measure ring;

(3) $\mathcal{F}$ has a strictly positive locally finite measure.

Proof. Since $\mathfrak{F}_{N}$ is a measure ring, (1) implies (2) by 3.4. Now assume (2), let $\varepsilon$ be the measure ring, and let $m$ be strictly positive and $\sigma$-finite on $\varepsilon$. 
Define $m^{*}$ on $\mathcal{F}$ by $m^{*}(u)=\sup \{m(e): e \leqq u\}$. We shall prove that $m^{*}$ is $\sigma$-additive. Let $\left\{u_{n}\right\}$ be a disjoint sequence with $\mathrm{V}_{n} u_{n}=u_{0}$. Clearly $\sum_{n} m^{*}\left(u_{n}\right) \leqq m^{*}\left(u_{0}\right)$. Now for any $e$ in $\mathcal{E}$ with $e \leqq u_{0}$, since $\mathcal{E}$ is an ideal we have $e \wedge u_{n} \in \mathcal{E}$ for each $n$, whence $m\left(e \wedge u_{n}\right) \leqq m^{*}\left(u_{0}\right)$. Thus $m(e)$ $=\sum_{n} m\left(e \wedge u_{n}\right) \leqq \sum_{n} m^{*}\left(u_{n}\right)$, and $m^{*}\left(u_{0}\right)=\sup m(e) \leqq \sum_{n} m^{*}\left(u_{n}\right)$. Evidently $m^{*}$ is strictly positive (because $\mathcal{E}$ is dense) and locally finite, so we have (3). Finally, assume (3). Let $m^{*}$ be strictly positive and locally finite. Then any nonzero $u$ contains a nonzero $e$ such that $m^{*}(e)<\infty$, and $m^{*}$ is strictly positive on $e \wedge \mathcal{F}$. Thus $e \in \mathcal{F}_{N}$, and $u \in \mathcal{F}_{N}^{\frac{1}{N}}$, proving (1).

We remark that the completeness assumption is superfluous in conjunction with condition (2), since according to 3.4 and 5.2 the compactification of a measure ring is automatically complete. An algebra satisfying conditions (1)-(3) of 5.3 will be called a spectral algebra. Clearly any measure ring gives rise to a spectral algebra, its compactification, and every spectral algebra gives rise to a measure ring, its normal ideal. The proof that this establishes a one to one correspondence is conveniently based on a lemma which will have another use in $\S 9$. In any Boolean ring $\mathcal{F}$, let us call an element $e$ Archimedean when any disjoint family in $e \wedge \mathcal{F}$ is at most countable. The set of Archimedean elements is clearly a $\sigma$-ideal $\mathscr{F}_{A}$, and $\mathscr{F}_{N} \subset \mathscr{F}_{A}$.

\section{Lemma 5.4. Any dense $\sigma$-ideal $\mathfrak{g}$ in $\mathcal{F}$ contains $\mathfrak{F}_{A}$.}

Proof. Let $u \in \mathcal{F}_{A}$. Choose a maximal disjoint family in $\mathscr{g} \cap(u \wedge \mathfrak{F})$. Then this is only a countable family $\left\{e_{n}\right\}$, and $\bigvee_{n} e_{n}=e_{0} \in \mathcal{g}$. Obviously $e_{0} \leqq u$, and by the maximality of $\left\{e_{n}\right\}$ and the denseness of $\mathscr{g}, e_{0}=u$, whence $u \in \mathscr{g}$.

Corollary 5.5. If $\mathcal{F}$ is a spectral algebra, then $\mathfrak{F}_{A}=\mathcal{F}_{N}$.

Corollary 5.6. For any measure ring $\mathcal{E}$, the image of $\mathcal{\varepsilon}$ in $\bar{\varepsilon}$ is $(\bar{\varepsilon})_{N}$.

THEOREM 5.7. There is a one to one correspondence $\& \leftrightarrow \mathcal{F}$ between measure rings $\mathcal{E}$ and spectral algebras $\mathcal{F}$, such that $\mathcal{E}$ is the normal ideal of $\mathcal{F}$ and $\mathcal{F}$ is the compactification of $\mathcal{E} ;$ further, $\mathfrak{R}(\mathcal{E})=\mathfrak{R}(\mathcal{E})$ and $\mathfrak{N}(\mathcal{F})$ are isometric.

Proof. 5.3 (2) and 5.6; for the last part, 4.4. Here "one to one" means, of course, up to an isomorphism.

I do not know whether a spectral algebra can have nonzero purely $\sigma$-additive measures. If not, then $\mathfrak{R}(\mathfrak{F})=\mathfrak{N}(\mathfrak{F})$. A partial answer to this question is given in 6.8 below.

6. The structure of spectral algebras. Because of the close relationship between measure rings and measure algebras, it is not at all surprising that Maharam's classification [11] of homogeneous measure algebras can be extended to include measure rings, and therefore also spectral algebras. We begin with some examples:

6.1. Let $S$ be a universe of power $\mathfrak{a}$, and let $\mathcal{F}_{S}$ be the algebra of all subsets of $S$. The measure associated with each single point of $S$ is a normal measure on $\mathfrak{F}_{S}$, and therefore $\mathfrak{F}_{S}$ is spectral. Such an algebra is atomic. Conversely, if 
$\mathcal{F}$ is atomic and $\mathfrak{a}$ is the number of atoms in $\mathfrak{F}$, then clearly $\mathfrak{F}$ is isomorphic to $\mathcal{F}_{S}$.

6.2. Any measure algebra is a spectral algebra. Maharam decomposes any measure algebra into an atomic part and principal ideals which are homogeneous, and classifies homogeneous measure algebras by an infinite cardinal parameter $\mathfrak{m}$ which we shall call the index; namely $\mathfrak{m}$ is the least cardinal number which is the power of a $\sigma$-basis of the algebra. The measure algebra of index $\boldsymbol{\aleph}_{0}$ is the Lebesgue measure algebra $F_{0}$ associated with a unit interval; that of index $\mathfrak{m}, \mathfrak{m} \geqq \boldsymbol{N}_{0}$, is the $\mathfrak{m}$-fold product of $\digamma_{0}$ with itself.

6.3. Let $\left\{\mathcal{F}_{\alpha}\right\}$ be a family of spectral algebras, $\alpha$ running through $A$. We define the direct union $\mathcal{F}=\oplus\left\{\mathcal{F}_{\alpha}\right\}$ as the algebra of all functions $e(\alpha)$ defined on $A$ with $e(\alpha) \in F_{\alpha}$ for each $\alpha$, the operations being defined pointwise. Then $\left\{F_{\alpha}\right\}$ can be regarded as a disjoint dense family of principal ideals in $\mathcal{F}$, and any normal measure on $\mathcal{F}_{\alpha}$ extends in the obvious way to a normal measure on $\mathcal{F}$. Therefore $\mathcal{F}$ is spectral. (From the other direction, to prove that $\mathcal{F}$ is the direct union of ideals $\left\{\mathcal{F}_{\alpha}\right\}$, it is enough to show the $\left\{\mathscr{F}_{\alpha}\right\}$ disjoint and their union dense in $\mathcal{F}_{\text {.) }}$ In particular, when $A$ has power $\mathfrak{n}$ and each $\mathcal{F}_{\alpha}$ is a homogeneous measure algebra of index $\mathfrak{m}$, we denote $\oplus\left\{\mathcal{F}_{\alpha}\right\}$ by $\mathcal{F}(\mathfrak{m}, \mathfrak{n})$; it can be regarded as a product of spectral algebras of types 6.1 and 6.2 .

LEMma 6.4. In any spectral algebra $\mathcal{F}$, every infinite maximal disjoint family of nonzero elements of the measure ideal $\mathfrak{F}_{N}$ of $\mathfrak{F}$ has the same power.

Proof. Every such family in a measure algebra has power $\boldsymbol{\aleph}_{0}$. Suppose now that $\left\{e_{\alpha}\right\}$ and $\left\{e_{\beta^{\prime}}\right\}$ are two such families in $\mathfrak{F}_{N}$ having powers $\mathfrak{n}$ and $\mathfrak{n}^{\prime}$ respectively. Then $\left\{e_{\alpha} \wedge e_{\beta^{\prime}}\right\}$ is again such a family except that some elements may be 0 . Let $\mathfrak{n}^{\prime \prime}$ be the number of nonzero elements in $\left\{e_{\alpha} \wedge e_{\beta^{\prime}}\right\}$. For each fixed $\alpha, e_{\alpha} \wedge \mathcal{F}$ is a measure algebra $\left(e_{\alpha}\right.$ being in $\left.\mathscr{F}_{N}\right)$, so that at most $\boldsymbol{\aleph}_{0}$ of the $\left\{e_{\alpha} \wedge e_{\beta^{\prime}}\right\}$ (for that $\alpha$ ) survive. Therefore $\mathfrak{n} \leqq \mathfrak{n}^{\prime \prime} \leqq \mathfrak{n} \cdot \boldsymbol{\aleph}_{0}=\mathfrak{n}$. Similarly $\mathfrak{n}^{\prime}=\mathfrak{n}^{\prime \prime}$, and the lemma is proved.

Except for the trivial finite atomic case (to which we assign a finite dimension) every spectral algebra $\mathfrak{F}$ has a disjoint family of the kind occurring in 6.4 , and its power uniquely defines the dimension $\mathfrak{n}$ of $\mathcal{F}$. The dimension of a direct union is clearly the sum of the dimensions of the component ideals, and therefore this terminology assigns dimension $\mathfrak{n}$ to the $\mathscr{F}(\mathfrak{m}, \mathfrak{n})$ of 6.3 .

We shall call a spectral algebra $\mathcal{F}$ homogeneous when every pair of nonzero principal ideals contained in $\mathcal{F}_{N}$ are isomorphic. When $\mathcal{F}$ is a measure algebra, this is equivalent to Maharam's definition, and therefore in a homogeneous spectral algebra $\mathcal{F}$, all principal ideals in $\mathfrak{F}_{N}$ are homogeneous measure algebras with the same index, which may also be called the index of $\mathcal{F}$.

Theorem 6.5. Any homogeneous spectral algebra $\mathfrak{F}$ with index $\mathfrak{m}$ and dimension $\mathfrak{n}$ is isomorphic to the algebra $\mathfrak{F}(\mathfrak{m}, \mathfrak{n})$ of 6.3 .

Proof. By hypothesis, we have a maximal disjoint family $\left\{e_{\alpha}\right\}$ in $\mathcal{F}_{N}$ 
with indices in a set $A$ of power $\mathfrak{n}$, each $\mathcal{F}_{\alpha}=e_{\alpha} \wedge \mathcal{F}$ being a homogeneous measure algebra of index $\mathfrak{m}$. The desired isomorphism of $\mathcal{F}$ with $\oplus\left\{\mathcal{F}_{\alpha}\right\}$ makes correspond to $u$ in $\mathcal{F}$ the function $e(\alpha)=u \wedge e_{\alpha}$.

Theorem 6.6. Any spectral algebra is the direct union of an atomic algebra and of homogeneous spectral algebras, one for each index.

Proof. Let $\mathfrak{F}_{A}=u_{A} \wedge \mathcal{F}$, where $u_{A}$ is the join of all atoms in $\mathscr{F}$. For each infinite cardinal $\mathfrak{m}$, let $\mathcal{F}_{\mathfrak{m}}=u_{\mathfrak{m}} \wedge \mathcal{F}$, where $u_{\mathfrak{m}}$ is the join of all $e$ in $\mathcal{F}_{N}$ such that $e \wedge \mathcal{F}$ is a homogeneous measure algebra of index $\mathrm{m}$. It is clear that $\mathfrak{F}_{\boldsymbol{A}}$ is atomic. Also $\mathcal{F}_{\mathfrak{m}}$ is homogeneous of index $\mathfrak{m}$, for if $e \in \mathcal{F}_{\mathfrak{m}} \cap \mathcal{F}_{N}$, then $e$ is a countable join $\bigvee_{n} e_{n}$ in which each $e_{n} \wedge \mathcal{F}$ is a homogeneous measure algebra of index $\mathfrak{m}$, whence $e \wedge F$ is one also. It follows that $\left\{\mathcal{F}_{A}, \mathscr{F}_{\mathfrak{m}}\right\}$ is a disjoint family. Finally, the union $\mathfrak{g}$ of $\mathcal{F}_{A}$ and $\left\{\mathcal{F}_{\mathfrak{m}}\right\}$ is dense, for any nonzero $u$ contains a nonzero $e$ in $\mathcal{F}_{N}$ (F $\mathcal{F}$ being spectral), and then $e \wedge \mathcal{F}$ is a measure algebra, which by Maharam's decomposition has some nonzero part in $g$.

Corollary 6.7. The structure of a spectral algebra $F$ is completely determined by specifying (1) the number a of atoms (which may be any cardinal number) and (2) for each infinite cardinal $\mathfrak{m}$ the dimension $\mathfrak{n}(\mathfrak{m})$ of the homogeneous ideal of index $\mathfrak{m}$ (this may be 0 or any infinite cardinal). The dimension of $\mathcal{F}$ is $\mathfrak{a}+\sum_{\mathfrak{m}} \mathfrak{n}(\mathfrak{m})$.

If the dimension of a spectral algebra is not too large, we can use a theorem of Ulam to answer the question raised at the end of $\$ 5$.

THEOREM 6.8. If $\mathcal{F}$ is a spectral algebra whose dimension is less than every inaccessible cardinal, then every finite measure on $\mathfrak{F}$ is normal.

Proof. A cardinal $\mathfrak{p}$ is inaccessible if it is a limit cardinal and cannot be represented as a sum of fewer than $\mathfrak{p}$ cardinals each less than $\mathfrak{p}$. Ulam's theorem $[16$, Satz (A) $]$ is the special case of 6.8 in which $F$ is atomic. To prove the general case from the atomic case, let $\left\{e_{\alpha}\right\}$ be a maximal disjoint family of nonzero elements of $\mathcal{F}_{N}$. The power of the index class $\{\alpha\}=A$ is then $\mathfrak{n}$, the dimension of $\mathcal{F}$. Suppose there exists a nonzero purely $\sigma$-additive measure $m$ on $\mathcal{F}$ and define $m_{A}$ on the atomic algebra of all subsets of $A$ by $m_{A}(S)$ $=m\left(\bigvee\left\{e_{\alpha}: \alpha \in S\right\}\right)$ for each $S \subset A$. Then $m_{A}$ is $\sigma$-additive, $m_{A}(A)=m(1) \neq 0$, and $m_{A}$ vanishes on points of $A$ (because $m$ was purely $\sigma$-additive), contradicting Ulam's theorem.

7. Integration. The role of continuous functions on $\Omega$ is to act as operators on $\mathfrak{R}$. The agency which makes them do this is integration, but very little integration theory is actually needed. There is no need to consider measurable functions because every Baire measurable function is equal except on a first category set to a continuous function, and the measures under consideration vanish on first category sets. There is no need for unbounded functions as long as we deal only with bounded operators. Rather than invoke the heavy 
forces of measure theory which are applicable, we shall at small cost make a miniature integration theory just adequate for our purposes. It is based on the following more general lemma which is used again in $\$ 10$.

LEMMA 7.1. Given an isomorphism $u \rightarrow \tilde{u}$ of a Boolean algebra $\mathcal{F}$ into the $L$-decomposition of a Banach space $\mathfrak{X}$, there exists a unique norm preserving ring isomorphism $f \rightarrow \tilde{f}$ of $\mathfrak{E}(\Omega)$ onto the uniformly closed operator ring on $\mathfrak{X}$ generated by $\{\tilde{u}: u \in \mathcal{F}\}$, such that $\tilde{f}_{u}=\tilde{u}$ for every characteristic function $f_{u}$ in $\mathfrak{S}(\Omega)$.

Proof. Extend the correspondence $f_{u} \rightarrow \tilde{u}$ by linearity to step functions in (5. The crux of the lemma is that this extension is norm preserving, which is proved by a straightforward argument like the one used for the corresponding situation in Hilbert space. Since step functions are dense in $\mathfrak{E}$, the continuous extension to all of $\mathfrak{C}$ is possible, unique, and norm preserving. The relation $(f g)^{-}=\tilde{f} \tilde{g}$ is clear when $f$ and $g$ are characteristic functions, and thence follows easily for all $f$ and $g$.

The application of 7.1 to $\bar{\varepsilon}$ with $\mathfrak{X}=\mathfrak{R}(\mathcal{E})$ depends on the correspondence $u \rightarrow \tilde{u}$ being an isomorphism. It is a $\sigma$-homomorphism, but for it to be one to one requires that enough measures on $\varepsilon$ exist. We shall call $\varepsilon$ regular when for every nonzero $e$ in $\mathcal{E}$ there exists $m$ in $\mathbb{R}$ with $m(e) \neq 0$ (equivalently, $\mathbb{R}$ separates elements of $\varepsilon$ ). Of course measure rings and spectral algebras are regular. Any nonregular $\mathcal{E}$ can be replaced by a regular one with the same $\mathfrak{R}$ by taking out the $\sigma$-ideal where all measures vanish.

COROLLARY 7.2. If $\mathcal{E}$ is a regular $\sigma$-ring, then there exists a unique normpreserving ring isomorphism $f \rightarrow \tilde{f}$ of $\mathbb{S}(\Omega)$ into the operator algebra of $\&$, such that $\tilde{f}_{u}=\tilde{u}$ for every $u$ in $\bar{\varepsilon}$.

We shall denote by $R$ the ring of operators $\tilde{f}$ arising from $\mathfrak{E}$. We need to know for a given measure $m$ what measures are of the form $\tilde{f} m$ for some $\tilde{f}$ in $R$. This is evidently a question of Radon-Nikodym type, or from another point of view, a question of spectral representation.

It is easy to see that $\tilde{f} m$ is not merely absolutely continuous with respect to $m$, but has the stronger property of being $m$-bounded, which means satisfying a Lipschitz condition $|\tilde{f} m| \leqq k|m|$, where in this case $k=\|f\|$.

LEMma 7.3. If $m$ is normal and $m^{\prime}$ is $m$-bounded, then $m^{\prime}=\tilde{f} m$ for some $f$ in ( .

Proof. The spectral theory for functions $f$ in $\mathbb{E}$ is as follows. For each real $\lambda,\{s: f(s) \leqq \lambda\}$ has an open closed interior $u_{\lambda} \in \bar{\varepsilon}$. The correspondence $\lambda \rightarrow u_{\lambda}$ has the properties (1) $\lambda<\mu$ implies $u_{\lambda} \leqq u_{\mu}$ and (2) $u_{\lambda_{0}}=0, u_{\lambda_{1}}=1$, where $\lambda_{0}=-\|f\|$ and $\lambda_{1}=\|f\|$. Conversely, given any spectral family $\lambda \rightarrow u_{\lambda}$ satisfying (1) and (2) (for some $\lambda_{0}$ and $\lambda_{1}$ ), then $f(s)=\inf \left\{\lambda: s \in u_{\lambda}\right\}$ defines a function in $\mathfrak{S}$, and the correspondence so established between functions and spectral families is one to one.

In terms of the spectral family of $f$ the distinguishing property of $f m$ is 
that $\tilde{f} m \leqq \lambda m$ on $u_{\lambda} \wedge \mathcal{E}$ and $\tilde{f} m \geqq \lambda m$ on $\left(1-u_{\lambda}\right) \wedge \mathcal{E}$. We shall prove that $\tilde{f} m$ is the only measure with this property. Suppose $m^{\prime}$ satisfies $m^{\prime} \leqq \lambda m$ on $u_{\lambda} \wedge \varepsilon$ for every $\lambda$. If in particular $f$ is a step function, it is clear that $m^{\prime} \leqq \tilde{f} m$. But in any case there is a step function $g$ such that $f \leqq g \leqq f+\epsilon$, so we have $m^{\prime} \leqq \tilde{f} m$ $+\epsilon m$ for every positive $\epsilon$, or $m^{\prime} \leqq \tilde{f} m$. Similarly $m^{\prime} \geqq \lambda m$ on $\left(1-u_{\lambda}\right) \wedge \mathcal{E}$ leads to $m^{\prime} \geqq \tilde{f} m$.

Finally, let $m$ be normal, and let $m^{\prime}$ be $m$-bounded, say $\left|m^{\prime}\right| \leqq k|m|$. For each $\lambda$ let $u_{\lambda}$ be the support of the negative variation of $m^{\prime}-\lambda m$, an open closed set since $m^{\prime}-\lambda m$ is normal. Clearly (1) and (2) are satisfied with $\lambda_{0}=-k$ and $\lambda_{1}=k$. Hence by the preceding paragraph $m^{\prime}=\tilde{f} m$, where $f$ is the function with $\lambda \rightarrow u_{\lambda}$ for its spectral family.

LEMma 7.4. The $m$-bounded measures are dense in the L-subspace of measures absolutely continuous with respect to $m$.

Proof. Let $m^{\prime} \ll m$; we can assume $m \geqq 0$ and $m^{\prime} \geqq 0$. Define $u_{\lambda}$ for each $\lambda$ as in the preceding proof, and let $u_{\infty}=\bigvee_{n=1}^{\infty} u_{n}$. Then $m^{\prime} \geqq n m$ on $\left(1-u_{\infty}\right) \wedge \varepsilon$ for every positive integer $n$, and hence $m\left(1-u_{\infty}\right)=0$. Since $m^{\prime} \ll m, m^{\prime}\left(1-u_{\infty}\right)$ $=0$. Thus $m^{\prime}=\tilde{u}_{\infty} m^{\prime}=\lim _{n} \tilde{u}_{n} m^{\prime}$. Since $\tilde{u}_{n} m^{\prime}$ is $m$-bounded for every $n$, this proves the lemma.

We shall make $f$ in $\mathbb{S}$ act also as a functional on $\mathfrak{R}$ by means of the $u n i$ versal integration functional which we now define. When we regard $\mathcal{E}$ as a Moore-Smith index set using its upward order, each $m$ in $\&$ becomes a net of real numbers, in fact a convergent net, because of the existence of containers. Define $\int m$ as $\lim _{e} m(e)$. Then $\int$ is a linear functional of norm 1. For $f$ in 5 the composition $\int \tilde{f}$ of $\int$ with $\tilde{f}$ is a functional on \&. Instead of $\int \tilde{f}_{u}$ we shall write $\int_{u}$, when $f_{u}$ is a characteristic function.

Lemma 7.5. If $\mathcal{E}$ is a regular $\sigma$-ring, then the correspondence $f \rightarrow \int \tilde{f}$ is an isometry of $\mathbb{S}(\Omega)$ into $\Omega^{*}$ such that $\int_{e} m=m(e)$ for all $e$ in $\mathcal{E}$.

Proof. For any $m$ let $e$ be a container for $\tilde{f} m$. Then

$$
\left|\int \tilde{f} m\right|=|\tilde{f} m(e)| \leqq\|\tilde{f} m\| \leqq\|f\|\|m\|,
$$

whence $\left\|\int \tilde{f}\right\| \leqq\|f\|$. To prove the norms are equal, it is sufficient to consider step functions, which are dense. Let $f=\sum_{i=1}^{n} \lambda_{i} f_{u_{i}}$, and assume that $\max _{i}\left|\lambda_{i}\right|$ $=\lambda_{1}>0$. Since $\mathcal{E}$ is regular, there exists $m$ with support contained in $u_{1}$. Then $\left\|\int \tilde{f}\right\|\|m\| \geqq\left|\int \tilde{f}\right| m||=\lambda_{1}|m|\left(e_{1}\right)=\|f\|\|m\|\left(e_{1} \leqq u_{1}\right.$ being a container $)$, whence $\left\|\int \tilde{f}\right\| \geqq\|f\|$.

The following adjunct to 7.1 will be used in $\$ 10$.

Lemma 7.6. Extending the notation and hypothesis of 7.1, let $x \in \mathfrak{X}$ and let $m_{0}$ be the measure on $\mathcal{F}$ defined by $m_{0}(u)=\|\tilde{u} x\|$. Then $\|\tilde{f} x\|=\left\|\tilde{f} m_{0}\right\|$ for every $f \in \mathbb{S}(\Omega)$. 
Proof. For $f$ a characteristic function in $\mathbb{E}$, the conclusion is immediate from the definition of $m_{0}$. Thence it follows easily for step functions in $\mathfrak{E}$, because $\mathcal{F}$ acts in $\mathfrak{X}$ as $L$-projections. Finally, for an arbitrary $f$ in $\mathbb{E}$ and any $\epsilon>0$, find a step function $f_{\epsilon}$ in $\mathbb{E}$ such that $\left\|f-f_{\epsilon}\right\|<\epsilon\|x\|$. Then we have

$$
\left|\|\tilde{f} x\|-\left\|\tilde{f}_{\epsilon} x\right\|\right| \leqq\left\|\tilde{f} x-\tilde{f}_{\epsilon} x\right\| \leqq\left\|f-f_{\epsilon}\right\| \cdot\|x\|<\epsilon,
$$

and similar inequalities with $x$ replaced by $m_{0}$. Since $\left\|\tilde{f}_{\epsilon} x\right\|=\left\|\tilde{f}_{\epsilon} m_{0}\right\|$ it follows that \|\|$\tilde{f} x\|-\| \tilde{f} m_{0} \| \mid<2 \epsilon$, whence $\|\tilde{f} x\|=\left\|\tilde{f} m_{0}\right\|$.

8. Classical $\sigma$-rings. The mappings $u \rightarrow \tilde{u}$ of $\bar{\varepsilon}$ into the $L$-decomposition of $\mathfrak{R}$ and $f \rightarrow \int \tilde{f}$ of $\mathbb{C}$ into $\mathfrak{\Omega}^{*}$ suggest two questions: (1) The $L$-decomposition question in $\&$-to determine all $L$-projections; in particular, when are they all of the form $\tilde{u}$ ? (2) The conjugation question-to describe $\ell^{*}$, in particular when is it the set of all $\int \tilde{f}$ for $f \in \mathcal{C}$ ? We shall see that these questions are related to each other, and to the problem of representing $\&$ as $L^{1}$. This section deals only with finding those $\sigma$-rings for which $\Omega^{*}=\mathbb{5}$.

It is convenient to work in terms of operator rings on $\mathbb{R}$. This can be done by mapping $\mathfrak{R}^{*}$ into the operator algebra of $\mathfrak{R}$ much as we have done for $\mathfrak{\complement}$. In general, if $\mathcal{F}$ is a $\sigma$-complete algebra of projections of norm 1 in a Banach space $\mathfrak{X}$, there is a natural linear norm preserving map $x^{*} \rightarrow T_{x^{*}}$ of $\mathfrak{X}^{*}$ into the space of bounded linear transformations of $\mathfrak{X}$ into $\mathfrak{R}(\mathcal{F})$, defined by $T_{x^{*}} x(e)=x^{*}(\tilde{e} x)$, where $\tilde{e} \in F, x \in \mathfrak{X}, x^{*} \in \mathfrak{X}^{*}$, and $T_{x^{*}} x$ is a measure on $\mathcal{F}$. If in particular $\mathcal{F}=\overline{\mathcal{E}}$ and $\mathfrak{X}=\mathfrak{R}(\mathcal{E})$, then this correspondence maps $\mathfrak{R}(\mathcal{E})^{*}$ into operators from $\mathcal{R}(\mathcal{E})$ to $\mathcal{R}(\overline{\mathcal{E}})$, but we can restrict $T_{x^{*}} m$ to $\mathcal{E}$ without change of norm, because a container $e$ for $m$ is a container for $T_{x}^{*} m$, and if $m$ is chosen so that $x^{*}(m)>\left\|x^{*}\right\|\|m\|-\epsilon$, then $\left\|T_{x^{*}} m\right\| \geqq T_{x^{*}} m(e)=x^{*}(m)>\left\|x^{*}\right\|\|m\|-\epsilon$. Let $\mathfrak{R}_{C}$ be the set of operators $T_{x^{*}}$ in $\mathbb{R}$ arising from $\mathfrak{R}^{*}$ in this way. For $f \in C$, $\int \tilde{f} \in \mathfrak{R}^{*}$, and we have by definition

$$
T_{\int \tilde{f}} m(e)=\int \tilde{f} \tilde{e} m=\int_{e} \tilde{f} m=\tilde{f} m(e),
$$

whence $T_{\int \tilde{f}}=\tilde{f}$. Thus $R \subset \Re_{C}$.

Theorem 8.1. Let $T$ be a bounded operator on $\mathfrak{R}$. Then the following conditions are equivalent:

(1) T commutes with $R$;

(2) $T \in R_{C}$;

(3) $T$ is absolutely continuous in the following sense: Tm $\ll m$ for every $m$ in \&.

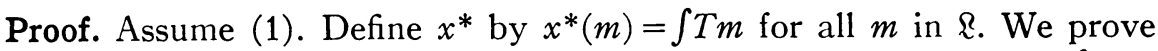
(2) by showing that $T=T_{x^{*}}$. But for any $e$ we have $T_{x^{*}} m(e)=x^{*}(\tilde{e} m)=\int T \tilde{e} m$ $=\int \tilde{e} T m=\int_{e} T m=T m(e)$, so $T_{x^{*}} m=T m$, and $T_{x^{*}}=T$.

Assume (2), and let $T=T_{x^{*}}, x^{*} \in \mathfrak{R}^{*}$. Then $\tilde{e} m=0$ implies $T m(e)=x^{*}(\tilde{e} m)$ $=0$ so $T m \ll m$ and we have (3). 
Finally, assume (3). To prove (1) it is enough to show that $T \tilde{e}=\widetilde{e} T$ for all $e$ in $\varepsilon$. To do this, let $m \in L$ and $e^{\prime} \in \mathcal{E}$. By hypothesis $T \tilde{e} m \ll \tilde{e} m$, and hence $T \tilde{e} m\left((1-e) \wedge e^{\prime}\right)=0$. Similarly $T(1-\tilde{e}) m\left(e \wedge e^{\prime}\right)=0$. Hence

$$
\begin{aligned}
T m\left(e^{\prime}\right) & =T \widetilde{e} m\left(e^{\prime}\right)+T(1-\tilde{e}) m\left(e^{\prime}\right) \\
& =T \tilde{e} m\left(e \wedge e^{\prime}\right)+T(1-\tilde{e}) m\left((1-e) \wedge e^{\prime}\right) \\
& =\tilde{e} T \tilde{e} m\left(e^{\prime}\right)+(1-\tilde{e}) T(1-\tilde{e}) m\left(e^{\prime}\right) .
\end{aligned}
$$

Abstracting the operators and simplifying gives $\tilde{e} T+T \tilde{e}=2 \tilde{e} T \tilde{e}$, and then pre- and post-multiplication by $\tilde{e}$ gives $\tilde{e} T=\tilde{e} T \tilde{e}$ and $T \widetilde{e}=\tilde{e} T \tilde{e}$ respectively. Thus $\tilde{e} T=T \tilde{e}$ and (1) is proved.

COROLlaRY 8.2. The following conditions for a $\sigma$-ring $\&$ are equivalent:

(1) $R$ is a maximal Abelian ring of operators;

(2) $R=R_{C}$;

(3) R contains all absolutely continuous operators.

We shall call a regular $\sigma$-ring $\mathcal{E}$ classical when $\varepsilon$ satisfies the conditions (1)-(3) of 8.2. That measure rings are classical (8.4) amounts to a restatement of the classical fact that the conjugate of $L^{1}$ is $L^{\infty}$ (see the remarks following 5.1).

THEOREM 8.3. The L-decomposition of a classical $\sigma$-ring $\&$ consists of the projections $\tilde{u}$ for $u \in \bar{\varepsilon}$.

Proof. By 2.2 any $L$-projection commutes with $R$, and therefore belongs to $R$, since $\mathcal{E}$ is classical. The corresponding function in $\mathbb{E}$ must be a characteristic function, because $f \rightarrow \tilde{f}$ is a ring isomorphism.

We shall see (9.8) that this condition is also sufficient for $\varepsilon$ to be classical. The rest of this section is devoted to determining as far as possible what $\sigma$-rings are classical.

TheOREM 8.4. Measure rings are classical.

Proof. Let $\varepsilon$ be a measure ring, and let $T$ be absolutely continuous. We shall prove that $T \in R$. For any $m, T m$ is $m$-bounded, because (using 8.1) $|T m|(e)=\|\tilde{e} T m\|=\|T \tilde{e} m\| \leqq\|T\| \cdot\|\tilde{e} m\|=\|T\| \cdot|m|(e)$. Since $\varepsilon$ is a measure ring, $m$ is normal, and by 7.3 there exists $f$ in $(5$ such that $T m=\tilde{f} m$. To avoid ambiguity we consider $f$ to be defined only on the support $e$ of $m$, this being sufficient to determine $\tilde{f} m$. Now for any $g$ in $\mathbb{5}$ we have $T \tilde{g} m=\tilde{g} T m=\tilde{g} \tilde{f} m$ $=\tilde{f} \tilde{g} m$. Since by $7.4\{\tilde{g} m\}$ is dense in $\tilde{e} R, T$ and $\tilde{f}$ are equal on $\widetilde{e}$. It follows that the functions resulting from different measures agree on the overlap of their domains, and therefore that there is one continuous $f$ defined on $\Omega$ and bounded by $\|T\|$ which extends them all. Clearly $T=\tilde{f}$.

COROLLARY 8.5. If $\mathfrak{F}$ is a spectral algebra, then the L-decomposition of $\mathfrak{R}(\mathfrak{F})$ is the set of projections $\tilde{u}$ for $u \in \mathcal{F}$. 
Proof. 5.7, 8.3 and 8.4.

CoRollary 8.6. If $\mathcal{F}$ is a spectral algebra, then $\mathfrak{N}(\mathcal{F}) *$ is representable as $(\widetilde{\Omega}(\bar{\Omega}), \bar{\Omega}$ being the Stone space of $\mathcal{F}$.

Proof. By $5.7 \mathfrak{R}(\mathfrak{F})$ is the same as $\mathscr{R}\left(\mathfrak{F}_{N}\right)$. By $8.4 \mathscr{F}_{N}$ is classical, that is $\mathfrak{R}\left(\mathcal{F}_{N}\right) *$ is representable as $\mathfrak{S}\left(\overline{\mathfrak{F}}_{N}\right)$. But $\overline{\mathfrak{F}}_{N}=\mathfrak{F}$ by 3.4 .

Lemma 8.7. If $\mathcal{E}$ is classical, then every measure on $\mathcal{E}$ is normal.

Proof. Suppose there exists on $\mathcal{E}$ a purely $\sigma$-additive measure $m$. By 8.3 the $L$-projection whose range consists of all measures absolutely continuous with respect to $m$ is $\tilde{u}$ for some $u$ in $\bar{\varepsilon}$. Now $u$ being open and the support $S_{m}$ of $m$ being nondense, there exists $e \neq 0$ in $\varepsilon$ contained in $u$ and disjoint from $S_{m}$. Then any nonzero measure $m^{\prime}$ whose support is contained in $e$ (they exist, since $\mathcal{E}$ is regular) belongs to $\tilde{u} R$, but is not absolutely continuous with respect to $m$, a contradiction.

Theorem 8.8. Every classical $\sigma$-ring $\mathcal{E}$ is isomorphic to a $\sigma$-ideal in a spectral algebra.

Proof. Let $\mathcal{F}$ be the compactification of the normal ideal of $\mathcal{E} ; \mathcal{F}$ is a spectral algebra. For each $e$ in $\mathcal{E}, e \wedge \varepsilon_{N}$ is a locally principal ideal in $\mathcal{E}_{N}$, to which corresponds a unique element of $\mathcal{F}$, the correspondence being an isomorphism of $\varepsilon$ into $F$ because, by 8.7 and the regularity of $\varepsilon, \varepsilon_{N}$ is dense in $\varepsilon$. Identifying $\mathcal{E}$ with its image, we then have $\mathcal{E}_{N}=\mathcal{F}_{N} \subset \mathcal{E} \subset \mathcal{F}$, and it remains to prove that $\mathcal{E}$ is an ideal, hence a $\sigma$-ideal. Every $m$ in $\mathcal{R}(\mathcal{E})$, being normal, has an extension $T m$ to a normal measure on $\mathcal{F}$, and $T$ is an isometry. Let $\phi$ be the natural continuous mapping of $U \mathcal{E}$ (a subset of the Stone space $\bar{\Omega}$ of $\mathcal{F}$ ) onto $\Omega=\Omega(\mathcal{E})$, so that each $f$ in $\subseteq(\Omega)$ leads to a function $f \phi$ (or more precisely its extension) in $\mathbb{E}(\bar{\Omega})$. Then it is easy to see that $\int(f \phi)^{-i} T m=\int \tilde{f} m$ for every $m$ in $\mathcal{R}(\mathcal{E})$. Now for any $h$ in $\mathbb{E}(\bar{\Omega}), \int \tilde{h} T m$ as a function of $m$ belongs to $\mathscr{R}(\mathcal{E})^{*}$. By hypothesis there exists $f$ in $\widetilde{C}(\Omega)$ such that $\int \tilde{h} T m=\int \tilde{f} m=\int(f \phi)^{\sim} T m$ for all $m$. It follows that $h=f \phi$, hence that the mapping $f \rightarrow f \phi$ gives all of $\mathfrak{E}(\bar{\Omega})$. This implies that $\phi$ is a homeomorphism, and therefore that $\varepsilon$ is an ideal.

Theorem 8.9. A $\sigma$-ring $\mathcal{E}$ is classical if and only if $\mathcal{E}$ is regular and boundedly complete, and every measure on $\mathcal{E}$ is normal.

Proof. The necessity of the conditions has been proved in 8.7 and 8.8 . Conversely, the last condition insures that $\mathscr{R}(\mathcal{E})$ and $\mathscr{R}\left(\mathcal{E}_{N}\right)$ are identical, and with the regularity that $\varepsilon_{N}$ is dense. Also, $\varepsilon$ being boundedly complete implies that $\bar{\varepsilon}$ is complete. Therefore $\bar{\varepsilon}$ is also the compactification of its dense $\sigma$-ideal $\varepsilon_{N}$ by 3.4 , and the function rings $\mathcal{E}$ associated with $\varepsilon$ and $\varepsilon_{N}$ are also identical. Since $\varepsilon_{N}$ is classical by $8.4, \varepsilon$ is also.

TheOREM 8.10. In any spectral algebra $\mathfrak{F}$ there exists a maximal classical $\sigma$-ideal $\mathfrak{F}_{C}$ such that a $\sigma$-ideal $\mathcal{E}$ in $\mathfrak{F}$ is classical if and only if $\mathcal{E} \subset \mathfrak{F}_{C}$. 
Proof. Let $\mathcal{F}_{C}$ be the $\sigma$-ideal generated by all classical $\sigma$-ideals in $F_{\text {. Let }}$ $\mathcal{E}$ be any $\sigma$-ideal contained in $\mathcal{F}_{C}$. Then $\mathcal{E}$ is boundedly complete and regular. Any measure $m$ in $\mathscr{R}(\mathcal{E})$ has a container $e$ in $\mathcal{E}$, and $e$ is a countable join $\bigvee_{n} e_{n}$ of elements $e_{n}$ belonging to classical $\sigma$-ideals $\varepsilon_{n}$. Then for each $n, \tilde{e}_{n} m$ is normal, whence $m$ is also normal. By $8.9 \&$ is classical, and the theorem is proved.

The question whether $\mathcal{F}_{C}$ can fail to be all of $\mathcal{F}$ is equivalent to the question of existence of purely $\sigma$-additive measures. From 6.8 we see that $\mathcal{F}_{C}=\mathcal{F}$ if the dimension of $\mathfrak{F}$ is not too large. In particular, assuming the continuum hypothesis, this is true of dimension $c$, the power of the continuum, and every $\sigma$-ideal of subsets of the line is classical.

9. The normal extension, and the $L$-decomposition of $\mathfrak{R}$. We turn now to the conjugation and $L$-decomposition problems for $\sigma$-rings which are not classical, and show that any $\sigma$-ring can be replaced by a measure ring by using the results of $\S 2$.

In this section $\mathcal{E}$ will be an arbitrary $\sigma$-ring, and $\mathcal{F}$ will be the $L$-decomposition of $\mathbb{R}=\mathfrak{R}(\mathcal{E})$. However, to conform with previous notation we shall regard $\mathcal{F}$ as an abstract spectral algebra and write $\tilde{u}$ for the $L$-projection represented by $u \in \mathcal{F}$. According to 7.1 we have a ring isomorphism $f \rightarrow \tilde{f}$ of $\mathbb{E}(\bar{\Omega})(\bar{\Omega}$ being the Stone space of $\mathcal{F}$ ) with the operator ring $\mathbb{R}_{L}$ generated by $\mathcal{F}$, which extends the isomorphism of $\mathbb{S}(\Omega)$ with $R \subset R_{L}$. As before $R_{C}$ is the space of operators arising from $\mathfrak{R}^{*}$, and we have shown in 8.1 that $R_{C}=\mathfrak{R}^{\prime}$, where' denotes the commutant.

LEMMA 9.1. $\mathbb{R}^{\prime}=\mathbb{R}^{\prime \prime}$.

Proof. Since $R$ is Abelian, $R \subset R^{\prime}$, whence $R^{\prime \prime} \subset R^{\prime}$. The reverse inclusion $\mathfrak{R}^{\prime} \subset \mathbb{R}^{\prime \prime}$ asserts that $\mathcal{R}^{\prime}$ is Abelian, and this is to be proved. Let $T_{1}$ and $T_{2}$ belong to $\mathbb{R}^{\prime}$, and let $m \in R$. Then, according to 7.1, $T_{1} m \ll m$ and $T_{2} m \ll m$, so the $L$-subspace of measures absolutely continuous with respect to $m$ is invariant under $T_{1}$ and $T_{2}$. Now this subspace is (isomorphic to) $\mathfrak{R}(a)$ where $Q=\varepsilon / \mathscr{g}_{m}$ is a measure algebra, hence classical. Thus, considered in this smaller context, $T_{1}$ and $T_{2}$ belong to $\mathcal{R}^{\prime}=R_{C}=R$, and they commute. In particular $T_{1} T_{2} m=T_{2} T_{1} m$. Since $m$ was arbitrary $T_{1} T_{2}=T_{2} T_{1}$ and $R^{\prime}$ is Abelian.

Corollary 9.2. $\mathfrak{R}_{C}=\mathbb{R}^{\prime}$ is a maximal Abelian operator ring.

Our strategy is as follows. We have $R \subset R_{L} \subset R_{L}^{\prime}$, whence $R_{L} \subset \mathbb{R}^{\prime}=R_{C}$. To show that $R_{L}=R_{C}$ it would, according to 9.1 , suffice to prove that $R_{L}$ is maximal Abelian. This is already known for classical $\sigma$-rings. Since it is a purely geometric Banach space property, it will follow in general as soon as we show that $\mathfrak{R}(\mathcal{E})$ for any $\mathcal{E}$ is always isomorphic as a Banach space to $\mathscr{R}$ on a measure ring. We shall accomplish this by showing that every measure on $\mathcal{E}$ can be uniquely extended without changing its norm to a normal measure on $\mathfrak{F}$. This will give an isometry between $\mathfrak{R}(\mathcal{E})$ and $\mathfrak{N}(\mathfrak{F})=\mathfrak{I}\left(\mathfrak{F}_{N}\right)$. The existence 
of the extension is easy to prove; the following lemmas are aimed at establishing the equality of the norms.

We shall call a $\sigma$-subring $\mathcal{E}$ of a spectral algebra $\mathcal{F}$ measure dense when for every $u$ in $\mathcal{F}_{N} u \wedge \mathcal{F}=u \wedge \mathcal{E}$.

LEMMA 9.3. In order for the restriction of normal measures on a spectral algebra $\mathcal{F}$ to a $\sigma$-subalgebra $\mathcal{E}$ to be norm preserving it is necessary and sufficient that $\&$ be measure dense.

Proof. Let $m$ be a normal measure on $\mathcal{F}$ with support $u$. For $e$ in $\mathcal{E}$ let $\beta e=e \wedge u$. Then $\beta$ is a $\sigma$-homomorphism of $\mathcal{E}$ into $u \wedge \mathcal{F}$. Moreover $m(\beta e)$ $=m(e)$, so the norm of the restriction of $m$ to $\mathcal{E}$ is the same as the norm of its restriction to $\beta(\mathcal{E})$, which is a $\sigma$-subring of $u \wedge \mathcal{F}$. The problem is thus reduced to the case where $\mathcal{F}$ is a measure algebra. Since the only measure dense subring of a measure algebra $\mathcal{F}$ is $\mathcal{F}$, the sufficiency is trivial. The necessity is proved by the same method as was used in 8.8. Suppose $m$ is strictly positive on $\mathcal{F}$. Then $m$ is strictly positive on $\mathcal{E}$, i.e. $\mathcal{E}$ is also a measure algebra, hence classical. Let $\phi$ be the natural map of $U_{\mathcal{E}} \subset \bar{\Omega}$ on $\Omega$. Then $\int \tilde{f} \rightarrow \int(f \phi)^{-}$is the transformation of $\mathfrak{R}(\mathcal{E})^{*}$ into $\mathfrak{R}(\mathcal{F})^{*}$ adjoint to the restriction operator mapping $\mathfrak{R}(\mathcal{F})$ into $\mathfrak{R}(\mathcal{E})$. For the latter to be norm preserving, hence one to one, $f \rightarrow f \phi$ must give all of $\mathbb{S}(\bar{\Omega})$, and this requires that $U \mathcal{E}=\bar{\Omega}$ and $\phi$ be a homeomorphism, that is $\mathcal{E}=\mathcal{F}$.

Lemma 9.4. Let $\mathfrak{F}$ be the L-decomposition of $\mathfrak{R}(\mathcal{E})$. Then $\mathcal{E}$ is measure dense in $F$.

Proof. For any measure $m$ in $\mathscr{R}(\mathcal{E})$, let $\mathscr{I}_{m}$ be the $\sigma$-ideal in $\mathcal{E}$ on which $|m|$ vanishes, and let $\alpha$ be the natural homomorphism of $\varepsilon$ onto $Q=\mathcal{E} / \mathscr{I}_{m}$. Further, let $\tilde{u}_{m}$ be the $L$-projection whose range is the measures absolutely continuous with respect to $m$. Then by $4.1 \tilde{u}_{m} \&$ is isomorphic to $R(Q)$, and $Q$ is classical (a measure algebra). For any $u \leqq u_{m}$ there is an $L$-projection in $\&(Q)$, and therefore by 8.3 an $\alpha e$ in $a$ such that in $\tilde{u}_{m} \& \tilde{e}=\tilde{u}$, that is $e \wedge u_{m}=u \wedge u_{m}$. This proves that $u_{m} \wedge \mathcal{E}=u_{m} \wedge \mathcal{F}$ for every $m$. Now the set of $u_{m}$ for $m \in \mathcal{R}$ is a dense $\sigma$-ideal, so by 5.4 and 5.5 it is $\mathfrak{F}_{N}$, and the lemma is proved.

Theorem 9.5. Let $\mathcal{E}$ be a $\sigma$-ring, $\mathcal{F}$ the L-decomposition of $\mathcal{R}(\mathcal{E})$. Then every measure in $\mathfrak{R}(\mathcal{E})$ has a unique extension to a normal measure on $\mathcal{F}$ with the same norm. The resulting correspondence is an isometry of $\mathfrak{R}(\mathcal{E})$ onto $\mathfrak{N}(\mathfrak{F})$.

Proof. Given $m$ in $\mathfrak{R}(\mathcal{E})$, define $m^{*}$ on $\mathcal{F}$ by $m^{*}(u)=\int \tilde{u} m$. It is clear that $m^{*}$ is additive and bounded. If $\left\{u_{\alpha}\right\}$ is an increasing net in $\mathcal{F}$ and $u_{0}=\mathrm{V}_{\alpha} u_{\alpha}$, then $\tilde{u}_{0} m=\lim _{\alpha} \tilde{u}_{\alpha} m$, whence using the continuity of $\int, m^{*}\left(u_{0}\right)=\lim _{\alpha} m^{*}\left(u_{\alpha}\right)$. Thus $m^{*}$ is a normal measure. The restriction of $m^{*}$ to $\varepsilon$ is $m$ by the definition of $\int$. Hence by 9.3 and $9.4\left\|m^{*}\right\|=\|m\|$ and every measure in $\mathfrak{N}(\mathcal{F})$ occurs as $m^{*}$. 
Corollary 9.6. For any L-space $\mathfrak{X}$, there exists a unique (up to isomorphism) spectral algebra $\mathfrak{F}$ such that $\mathfrak{X}$ is isometric to $\mathfrak{R}(\mathfrak{F})$.

Proof. The existence comes from 9.5, the uniqueness from 8.5 and 9.5.

Corollary 9.7. The L-decomposition of $\mathfrak{R}$ is a maximal Abelian Boolean algebra of projections, and $\mathfrak{R}_{L}$ is a maximal Abelian operator ring.

Corollary 9.8. $R_{L}=R_{C}$.

CoROllary 9.9. $\mathcal{E}$ is classical if and only if $\mathcal{E}$ is regular and every $L$-projection in $\mathfrak{R}$ is $\tilde{u}$ for some $u$ in $\bar{\varepsilon}$.

Corollary 9.10 (KakUtani). For every $\mathcal{E}, \mathfrak{R}(\mathcal{E})$ has a representation as $L^{1}$.

10. Banach space properties of $L$-spaces. As has already been remarked, 9.7 expresses a property of $\mathfrak{Z}$ with purely Banach space meaning. In this section we shall exploit this to obtain geometric facts about $L$-spaces: a Banach space characterization of them, and a description of their symmetries. Note also that in view of 9.6 the structure theory for spectral algebras in $\$ 6$ can be translated into a structure theory for $L$-spaces. By $L$-space we mean any Banach space which is isomorphic to $\mathfrak{N}(\mathfrak{F})$ for some spectral algebra $\mathcal{F}$.

We shall continue to use the notation of $\S 9$ in so far as it applies to any Banach space $\mathfrak{X}$. Namely $\mathcal{F}$ denotes the $L$-decomposition of $\mathfrak{X}, \bar{\Omega}$ the Stone space of $\mathfrak{F}, \tilde{f}$ the operator on $\mathfrak{X}$ corresponding to $f \in \mathcal{C}(\bar{\Omega})$. There is no longer any $\mathcal{E}$ in question. For any $x$ in $\mathfrak{X}$, the closure $B_{x}$ of $\mathbb{R}_{L} x=\{\tilde{f} x: f \in \mathcal{S}(\bar{\Omega})\}$ is called the cycle generated by $x$; it is the smallest closed subspace containing $x$ invariant under $\mathcal{F}$. The range $\mathfrak{X}_{x}$ of the $L$-projection $\tilde{u}_{x}$, where $u_{x}$ $=\Lambda\{u: \tilde{u} x=x\}$, called the $L$-support of $x$, is the smallest $L$-subspace containing $x$. Evidently $\mathfrak{Z}_{x} \subset \mathfrak{X}_{x}$.

THEOREM 10.1. $\mathfrak{X}$ is an L-space if and only if $乃_{x}=\mathfrak{X}_{x}$ for every $x$.

Proof. The necessity of the condition was proved in 7.3 and 7.4. To prove the sufficiency, choose a maximal family $\left\{x_{\alpha}\right\}$ in $\mathfrak{X}$ such that the corresponding $L$-supports $\left\{u_{\alpha}\right\}$ are disjoint. Define a family $\left\{m_{\alpha}\right\}$ of normal measures by $m_{\alpha}(u)=\left\|\tilde{u} x_{\alpha}\right\|$. For each $x$ of the form $\sum_{i=1}^{n} \tilde{f}_{i} x_{\alpha_{i}}$ define $T x$ as $\sum_{i=1}^{n} \tilde{f}_{i} m_{\alpha_{i}}$, where $f_{1}, \cdots, f_{n} \in \mathfrak{C}$. Then $T$ maps such elements with preservation of norm into $\mathfrak{R}(\mathfrak{F})$ (see 7.6). By hypothesis and the maximality of $\left\{x_{\alpha}\right\}$ such $x$ are dense in $\mathfrak{X}$, so the continuous extension of $T$ is an isometry of $\mathfrak{X}$ onto $\mathfrak{R}(\mathcal{F})$.

Lemma 10.2. Every cycle is the range of a projection of norm 1 which commutes with $\mathfrak{F}$.

Proof. Given $x$, we go down immediately to $\mathfrak{X}_{x}$, which means that we can assume at the start, without loss of generality, that $\mathfrak{X}_{x}=\mathfrak{X}$. Let $m_{0}$ be the measure on $\mathcal{F}$ defined by $m_{0}(u)=\|\tilde{u} x\|$. Because of our starting assumption the 
support of $m_{0}$ is 1 , and therefore by 7.6 and $7.4 \tilde{f} x \rightarrow \tilde{f} m_{0}$ is an isometry of a dense subspace of $\nexists_{x}$ onto a dense subspace of $\mathscr{R}(\mathcal{F})$, which we extend to an isometry of $B_{x}$ onto $\mathfrak{R}(\mathcal{F})$. Take the functional on $B_{x}$ corresponding to the functional $\int$ on $\mathfrak{R}(\mathcal{F})$ under this isometry and extend it to a functional $x^{*}$ of norm 1 on $\mathfrak{X}$, by the Hahn-Banach theorem. Then $x^{*}(\tilde{u} x)=\|\tilde{u} x\|$ for all $u$ in $\mathcal{F}$. Now given $y$ in $\mathfrak{X}$, define $m_{y}$, a measure on $\mathcal{F}$, by $m_{y}(u)=x^{*}(\tilde{u} y)$. A slight modification of the proof of 7.4 shows that those $y$ such that $m_{y}$ is $m_{0}$-bounded are dense. For such a $y$, by 7.3 there exists $f_{v}$ in $\mathbb{5}$ such that $m_{y}=\tilde{f}_{y} m_{0}$; define $P y$ as $\tilde{f}_{y} x$. If we prove that $P$ is continuous, then $P$ extends to all $y$ in $\mathfrak{X}$. Now if $m_{y} \geqq 0$, then $\left\|m_{y}\right\|=m_{y}(1)=x^{*}(y) \leqq\|y\|$, and in any case $1=u^{+} \vee u^{-}$where $\tilde{u}^{+} m_{y} \geqq 0$ and $\tilde{u}^{-} m_{y} \leqq 0$, and then $\|P y\|=\left\|m_{y}\right\|=\left\|\tilde{u}^{+} m_{y}\right\|+\left\|\tilde{u}^{-} m_{y}\right\| \leqq\left\|\tilde{u}^{+} y\right\|$ $+\left\|\tilde{u}^{-} y\right\|=\|y\|$. Thus $P$ extends uniquely to an operator of norm 1 . We shall show that $P$ is a projection. Since the range is contained in $B_{x}$, we need only show that $P$ acts as the identity on $\nexists_{x}$. This hinges on the identity $x^{*}(\tilde{u} \tilde{f} x)$ $=\int_{u} \tilde{f} m_{0}$, which is proved by climbing up from the trivial case where $f$ is a characteristic function. On the other hand $x^{*}(\tilde{u} \tilde{f} x)=\int_{u} \tilde{f}_{\tilde{f} x} m_{0}$ by definition. Hence, since $u$ is free, $f_{\tilde{f} x}=f$ and $P \tilde{f} x=\tilde{f}_{\tilde{f} x} x=\tilde{f} x$. Since continuity extends this to all $y$ in $3_{x}, P$ is a projection with range $\nexists_{x}$. Finally, to see that $P$ commutes with $\mathcal{F}$, let $u \in \mathcal{F}$ and let $y \in \mathfrak{X}$. Observe that $m_{\tilde{u} y}=\tilde{u} m_{y}$, whence $f_{\tilde{u} y}=f_{u} f_{y}$, and $P \tilde{u} y=\tilde{f}_{\tilde{u} y} x=\tilde{f}_{u} \tilde{f}_{y} x=\tilde{u} P y$.

Theorem 10.3. $\mathfrak{X}$ is an L-space if and only if the L-decomposition of $\mathfrak{X}$ is maximal as an Abelian family of projections of norm 1.

Proof. The necessity was noted in 9.7 . For the sufficiency, let $x \in \mathfrak{X}$, and let $P$ be the projection of 10.2 with $B_{x}$ for range. Then by hypothesis $P$ is an $L$-projection, and therefore its range $\oiint_{x}$ contains $\mathfrak{X}_{x}$. The result now follows from 10.1 .

Two groups of symmetries of $\mathfrak{N}(\mathfrak{F})$ are apparent. Let $G_{L}$ be the group of isometries belonging to $R_{L}=R$ : each $u$ in $\mathcal{F}$ gives rise to an involutory isometry $2 \tilde{u}-1$, and these are all of $G_{L}$. Let $G_{0}$ be the group of isometries arising from automorphisms of $\mathcal{F}$ : for each automorphism $\gamma$ of $F$, define $T_{\gamma}$ by $T_{\gamma} m(u)=m\left(\gamma^{-1} u\right)$.

LEMMA 10.4. $(\gamma u)^{\sim}=T_{\gamma} \tilde{u} T_{\gamma}^{-1}$.

Proof. We have $T_{\gamma} \tilde{u} T_{\gamma}^{-1} m\left(u^{\prime}\right)=\tilde{u} T_{\gamma}^{-1} m\left(\gamma^{-1} u^{\prime}\right)=T_{\gamma}^{-1} m\left(u \wedge \gamma^{-1} u^{\prime}\right)$ $=m\left(\gamma u \wedge u^{\prime}\right)=(\gamma u)^{\dot{*}} m\left(u^{\prime}\right)$.

TheOREM 10.5. Every isometry $T$ of $\mathfrak{N}(\mathcal{F})$ has a unique factorization $T=T_{\gamma} \tilde{f}$, where $\tilde{f} \in G_{L}$ and $T_{\gamma} \in G_{0}$.

Proof. Since $T$ is norm preserving, any $L$-projection $\tilde{u}$ is mapped by $T$ into an $L$-projection $T \tilde{u} T^{-1}$, and this correspondence determines an automorphism $\gamma$ of $\mathcal{F}$ by 8.3. Then using 10.4 we have for any $u T_{\gamma}^{-1} T \tilde{u}$ $=T_{\gamma}^{-1}\left(T \tilde{u} T^{-1}\right) T=T_{\gamma}^{-1}(\gamma u)^{\sim} T=T_{\gamma}^{-1}\left(T_{\gamma} \tilde{u} T_{\gamma}^{-1}\right) T=\tilde{u} T_{\gamma}^{-1} T$, and hence $T_{\gamma}^{-1} T$ 
commutes with the $L$-decomposition. By 9.7 $T_{\gamma}^{-1} T=\tilde{f} \in G_{L}$, and we have the factorization $T=T_{\gamma} \tilde{f}$. Since clearly $G_{L} \cap G_{0}=\{1\}$, the factorization is unique.

The order of the factors in 10.5 is not important. In fact, $G_{L}$ is a normal subgroup of the symmetry group, and $T_{\gamma} \tilde{f}=\tilde{g} T_{\gamma}$, where $\tilde{g}=T_{\gamma} \tilde{f} T_{\gamma}^{-1} \in G_{L}$.

It is interesting to note that symmetries in $G_{0}$ preserve the partial order of $\mathfrak{N}$, while the only symmetry in $G_{L}$ which does so is the identity. Thus $G_{0}$ is the group of symmetries of $\mathfrak{N}$ regarded as a partially ordered normed linear space. On the other hand $G_{L}$ is the group of symmetries which leave invariant all $L$-subspaces. The direct product decomposition of the symmetry group of $\mathfrak{R}$ expressed by 10.5 can therefore be regarded as a duality between the $L$-structure and the order structure of $\mathfrak{N}$.

11. Abstract $M$-spaces and applications to topology. According to the theorem of Riesz-Markov every bounded linear functional on $\mathbb{S}(\Sigma)$, where $\Sigma$ is a compact Hausdorff space, is integration with respect to a unique regular Borel measure on $\Sigma$ (or a unique Baire measure). In our terminology this represents $\mathbb{E}(\Sigma)^{*}$ by $\mathcal{R}(\mathcal{E})$, where $\mathcal{E}$ is the $\sigma$-algebra of Baire sets in $\Sigma$. So stated this theorem contains two sources of dissatisfaction. The first is the advent of $\sigma$-operations, which played no previous role in the topology of $\Sigma$, as do finite and infinite lattice operations without further distinctions of cardinality. The second more serious one is that the representation of $\mathbb{S}(\Sigma)^{*}$ as $\mathbb{R}(\mathcal{E})$ does not lend itself to conjugation a second time, since $\varepsilon$ is rarely classical. Both of these objections could be overcome if the representation could be given directly in terms of the appropriate spectral algebra, rather than in terms of Baire or Borel sets. Now the conjugation of $\mathfrak{R}(\mathfrak{F})$ where $\mathcal{F}$ is spectral can be regarded as the dual of the Riesz-Markov theorem, and it can be used to give a proof of the latter which brings in the Borel sets of $\Sigma$ only at the end. It also deduces Kakutani's representation for abstract $M$-spaces from the one for abstract $L$-spaces. We shall sketch the argument.

If $\mathfrak{M}$ is an abstract $M$-space, then [9, Theorem 15] $\mathfrak{M}^{*}$ is an abstract $L$-space, and therefore it is $\mathfrak{R}(\mathcal{F})$ for a certain uniquely determined spectral algebra $\mathcal{F}$. Thus by $8.6 \mathfrak{M}^{* *}=\mathfrak{R}(\mathfrak{F})^{*}$ is $\mathfrak{S}(\Omega)$, where $\Omega$ is the Stone space of $\mathcal{F}$. Now $\mathfrak{S}(\Omega)$ is again an abstract $M$-space and [9, footnote 15] the natural embedding of $\mathfrak{M}$ in $\mathfrak{M}^{* *}$ is a complete lattice isomorphism, so $\mathfrak{M}$ can be regarded as a closed sublattice of $\mathfrak{E}(\Omega)$. The representation of $\mathfrak{M}$ thus reduces to describing the sublattices of $\mathbb{E}(\Omega)$, a relatively easy problem in which the Boolean nature of $\Omega$ plays no part. We consider only the case where $\mathfrak{M}$ contains a unit, which in $\mathbb{S}(\Omega)$ must turn out to be the constant function 1 . If $\mathfrak{M}$ separates points, then $\mathfrak{M}=\mathfrak{C}(\Omega)$; this is essentially the Stone-Weierstrass theorem [14]. In general define points $s$ and $t$ in $\Omega$ to be equivalent if $f(s)=f(t)$ for all $f$ in $\mathfrak{M}$. Let $\Sigma$ be the set of equivalence classes associated with this relation, let $\phi: \Omega \rightarrow \Sigma$ map each $s$ into the equivalence class containing it, and give $\Sigma$ the strongest topology for which $\phi$ is continuous. Then $\Sigma$ is compact and Hausdorff, the functions on $\Sigma$ obtained by transferring to $\Sigma$ functions in $\mathfrak{M}$ 
are continuous, and they form a sublattice of $\mathbb{E}(\Sigma)$ separating points and containing 1 , hence all of $\mathbb{(}(\Sigma)$.

In particular, if $\mathfrak{M}$ was to begin with $\mathbb{C}\left(\Sigma^{\prime}\right)$, where $\Sigma^{\prime}$ is compact and Hausdorff, then $\Sigma$ and $\Sigma^{\prime}$ are homeomorphic because of the identity of $\mathbb{E}(\Sigma)$ and $\mathfrak{M}=\mathbb{C}\left(\Sigma^{\prime}\right)$, hence the relevance of what follows to the problem of conjugating $\mathbb{S}\left(\Sigma^{\prime}\right)$.

Since $\mathcal{F}$ is a complete algebra, the closure of every open set in $\Omega$ is open [15], and hence every Borel set differs by a first category set from an open closed set. The correspondence $\beta$ which takes each Borel set into its associated open closed set is a $\sigma$-homomorphism of the Borel field of Borel sets of $\Omega$ into $\mathcal{F}$. Now $\phi^{-1}$ is an isomorphism of the algebra of all subsets of $\Sigma$ into that of $\Omega$, taking open sets into open sets, hence Borel sets into Borel sets. Thus $\beta \phi^{-1}$ is a $\sigma$-homomorphism taking Borel sets of $\Sigma$ into $\mathcal{F}$ (actually a $\sigma$-isomorphism, but this is not needed). For any normal measure $m$ on $\mathcal{F}, \mu(E)$ $=m\left(\beta \phi^{-1} E\right)$ defines a Borel measure in $\Sigma$, and evidently

$$
\int \tilde{f} m=\int_{\Sigma} f \phi^{-1} d \mu
$$

so that $m$ and $\mu$ give the same functional on $\mathfrak{M}$ by integration over $\Omega$ and $\Sigma$ respectively. We shall show that $\mu$ is a regular Borel measure. This means that for any Borel set $E$ in $\Sigma$ and any $\epsilon>0$, there exist a closed set $C$ and an open set $U$ with $B \subset E \subset U$ such that $|\mu|(U-C)<\epsilon$, or equivalently that for any open set $U \lim _{\alpha} \mu\left(U_{\alpha}\right)=\mu(U)$, where $\left\{U_{\alpha}\right\}$ is the net of open sets with $\bar{U}_{\alpha} \subset U$ directed by inclusion. By the definition of $\mu$ and the normality of $m$ we need only show that $\mathrm{V}_{\alpha} \beta \phi^{-1}\left(U_{\alpha}\right)=\beta \phi^{-1}(U)$. The truth of this rests only on the fact that $U=\mathrm{U}_{\alpha} U_{\alpha}$ and the identity for open sets $\beta \phi^{-1}(U)=\phi^{-1}(U)^{-}$. On one hand we have for each $\alpha \phi^{-1}\left(U_{\alpha}\right)^{-} C \phi^{-1}(U)^{-}$, whence $\bigvee_{\alpha} \beta \phi^{-1}\left(U_{\alpha}\right)$ $\leqq \beta \phi^{-1}(U)$. On the other hand if $u \in \mathcal{F}$ and $\beta \phi^{-1}\left(U_{\alpha}\right) \leqq u$ for all $\alpha$, then $\phi^{-1}(U)=\bigcup_{\alpha} \phi^{-1}\left(U_{\alpha}\right) \subset \bigcup_{\alpha} \phi^{-1}\left(U_{\alpha}\right)-\subset u$, and since $u$ is closed $\beta \phi^{-1}(U)=\phi^{-1}(U)^{-}$ $\leqq u$. Thus $\beta \phi^{-1}(U) \leqq \mathrm{V}_{\alpha} \beta \phi^{-1}\left(U_{\alpha}\right)$, and we have shown that $\mu$ is regular.

The uniqueness of a regular Borel measure to represent a given functional is straight-forward and need not be repeated (see for example [9, p. 1011]).

\section{REFERENCES}

1. W. G. Bade, On Boolean algebras of projections and algebras of operators, Trans. Amer. Math. Soc. vol. 80 (1955) pp. 345-360.

2. G. Birkhoff, Dependent probabilities and the space L, Proc. Nat. Acad. Sci. U.S.A. vol. 24 (1938) pp. 154-159.

3. - Lattice theory, Amer. Math. Soc. Colloquium Publications, vol. 25, 1948.

4. J. Dixmier, Sur certains espaces considérés par M. H. Stone, Summa Brasil. Math. vol. 2 (1951) pp. 151-182.

5. R. E. Fullerton, A characterization of L-spaces, Fund. Math. vol. 38 (1951) pp. 127-136.

6. P. R. Halmos, Introduction to Hilbert space, New York, Chelsea, 1951. 
7. L. J. Heider, $\sigma$-additive measures on abstract $\sigma$-rings, Bull. Amer. Math. Soc. vol. 63 (1957) Abstract 431.

8. S. Kakutani, Concrete representations of abstract $L$-spaces and the mean ergodic theorem, Ann. of Math. vol. 42 (1941) pp. 523-537.

9. - Concrete representation of abstract M-spaces, Ann. of Math. vol. 42 (1941) pp. 994-1024.

10. L. H. Loomis, On the representation of $\sigma$-complete Boolean algebras, Bull. Amer. Math. Soc. vol. 53 (1947) pp. 757-760.

11. D. Maharam, Homogeneous measure algebras, Proc. Nat. Acad. Sci. U.S.A. vol. 28 (1942) pp. 108-111.

12. I. E. Segal, Equivalence of measure spaces, Amer. J. Math. vol. 73 (1951) pp. 275-313.

13. M. H. Stone, Applications of the theory of Boolean rings to general topology, Trans. Amer. Math. Soc. vol. 41 (1937) pp. 375-481.

14. - The generalized Weierstrass approximation theorem, Math. Mag. vol. 21 (1948) pp. 167-184, 237-254.

15. - Boundedness properties in function lattices, Canad. J. Math. vol. 1 (1949) pp. 176-186.

16. S. Ulam, Zur Masstheorie in der allgemeinen Mengenlehre, Fund. Math. vol. 16 (1930) pp. 140-150.

WESLEYAN UNIVERSITY, Middletown, ConNecticut

HARVARD UNIVERSITY,

Cambridge, Massachusetts 